University of Tennessee Health Science Center

UTHSC Digital Commons

\title{
Bevacizumab Effect on Topotecan Pharmacokinetics in a Murine Orthotopic Rhabdomyosarcoma Xenograft Model
}

\author{
Zaifang Huang \\ University of Tennessee Health Science Center
}

Follow this and additional works at: https://dc.uthsc.edu/dissertations

Part of the Pharmacy and Pharmaceutical Sciences Commons

\section{Recommended Citation}

Huang, Zaifang , "Bevacizumab Effect on Topotecan Pharmacokinetics in a Murine Orthotopic Rhabdomyosarcoma Xenograft Model" (2011). Theses and Dissertations (ETD). Paper 120. http://dx.doi.org/10.21007/etd.cghs.2011.0143. 


\title{
Bevacizumab Effect on Topotecan Pharmacokinetics in a Murine Orthotopic Rhabdomyosarcoma Xenograft Model
}

\author{
Abstract \\ Increasing evidence suggests that inhibition of vascular endothelial growth factor (VEGF) can transiently \\ normalize tumor vasculature, thereby improving delivery of systemic chemotherapy. Bevacizumab (BEV), \\ an anti-VEGF antibody, has been shown to transiently normalize tumor vasculature by increasing tumor \\ vessel maturity, decreasing tumor vessel permeability, and increasing tumor oxygenation in an Rh30 \\ orthotopic rhabdomyosarcoma xenograft model. However, the effects of BEV on the pharmacokinetics of \\ TPT and the antitumor activity of TPT have not been evaluated. This study aimed to investigate the effect \\ of BEV on TPT systemic and tumoral pharmacokinetics and to determine how these changes affect the \\ efficacy of TPT in the Rh30 mouse model. Mice bearing Rh30 orthotopic xenografts were treated with \\ BEV alone $(5 \mathrm{mg} / \mathrm{kg})$, TPT alone $(2 \mathrm{mg} / \mathrm{kg}$ ) or a combination of the two administered intravenously with \\ different schedules. The pharmacokinetics of TPT, including TPT intratumoral penetration, as well as the \\ efficacy of the monotherapy and combination therapy were evaluated. Population pharmacokinetic \\ modeling and covariate analysis of TPT pharmacokinetics were performed using the maximal likelihood \\ expectation maximization (MLEM) method in ADAPT 5 to predict the plasma and tumor concentration- \\ time profile, to estimate the pharmacokinetic parameters of individual mouse and mice population, and to \\ evaluate the effect of BEV on TPT systemic and tumoral pharmacokinetics. Tumor penetration was \\ assessed by the tumor-to-plasma ratio of area under concentration-time curve (AUC). Tumor volume \\ before and after the treatment were measured to evaluate the antitumor activity of the treatment regimen, \\ and to assess the effect of BEV on the antitumor activity of TPT in Rh30 xenografts. Covariate analysis \\ showed a single dose of BEV was associated with the increased systemic elimination rate and clearance \\ of TPT. Furthermore, a single dose BEV had a time-dependent effect on the tumor elimination rate of TPT. \\ The elimination rate of TPT from tumor compartment increased when it was given 1 day after a single \\ dose of BEV and gradually decreased to control level when TPT was given 3 days and 7 days after a \\ single dose of BEV. Multiple doses of BEV had no effect on TPT pharmacokinetics. TPT penetration was \\ not altered after administering multiple doses of BEV, but a single dose of BEV produced a trend in \\ changes of TPT penetration. Tumor efficacy was not dependent on the schedule of BEV and TPT. TPT \\ significantly enhanced the antitumor activity of combination therapy while pre-treatment of BEV did not \\ alter the antitumor activity of TPT. Tumor efficacy in MDBT groups was mainly due to the multiple doses \\ of BEV and the antitumor activity of TPT was diminished. The present work provides crucial insights into \\ the effect of coadministration of BEV on the pharmacokinetic changes and antitumor activity of TPT. The \\ increased TPT systemic elimination and clearance after single dose of BEV treatment may be due to the \\ altered renal clearance by VEGF. The increased TPT elimination from tumor tissue after 1 day pre- \\ treatment of BEV may be caused by a normalization of tumor vasculature. The overall effect of BEV on \\ TPT pharmacokinetics as well as TPT penetration is determined by the net balance of the pharmacologic \\ changes of tumor microenvironment by BEV. And the antitumor activity of combination is determined by \\ the balance between angiogenesis inhibition-induced tumor cell starvation and the tumor cytotoxicity due \\ to the exposure to cytotoxic drugs. This study highlights the complexity of pharmacokinetic (PK) and \\ pharmacodynamic (PD) interaction that may take place when antiangiogenic agent and cytotoxic drug are \\ combined and cautions that more consideration and mechanistic investigation should be made before \\ using a combination of anti-angiogenic agents with cytotoxic drugs for cancer treatment.
}

\section{Document Type}

Thesis

\section{Degree Name}

Master of Science (MS) 


\section{Program}

Pharmaceutical Science

Research Advisor

Clinton F. Stewart, Pharm.D.

\section{Keywords}

Bevacizumab, Pharmacokinetics, Rhabdomyosarcoma, Topotecan

\section{Subject Categories}

Medicine and Health Sciences | Pharmacy and Pharmaceutical Sciences 
BEVACIZUMAB EFFECT ON TOPOTECAN PHARMACOKINETICS IN A MURINE ORTHOTOPIC RHABDOMYOSARCOMA XENOGRAFT MODEL

\author{
A Thesis \\ Presented for \\ The Graduate Studies Council \\ The University of Tennessee \\ Health Science Center
}

\author{
In Partial Fulfillment \\ Of the Requirements for the Degree \\ Master of Science \\ From The University of Tennessee
}

By

Zaifang Huang

December 2011 
Copyright (C) 2011 by Zaifang Huang. All rights reserved. 


\section{ACKNOWLEDGEMENTS}

I would like to thank my mentor, Dr. Clinton F. Stewart, for the opportunity to learn from his vast knowledge and work in his laboratory. I also would like to express my appreciation for his help, guidance, suggestion and encouragement throughout my study, research and writing of this thesis. He is an incredible good mentor and career guider.

My sincere gratitude is also extended to the other members of my thesis committee: Drs. John C. Panetta and Bernd Meibohm for serving on my committee and their excellent suggestion, comments and guidance for this project. Especially, I want to express my deepest gratitude to Dr. John C. Panetta for helping me with the population modeling of this project. Without his help, I cannot accomplish this part of the work. I also would like to thank Dr. Andrew M. Davidoff for providing the Rh30 cell line used in this study.

In addition, I would like to thank to all the members and friends in the Stewart laboratory. I would like to acknowledge my supervisor Dr. Stacy Throm and co-worker Dr. Fan Wang for their patience, guidance and support during the project progress and writing of the thesis. Thanks to the former post-docs Dr. Michael Tagen, Angel M. Carcaboso and the former supervisor Laura Miller for their constant support and help. I'm also grateful to co-workers and friends Dr. Feng Bai, Dr. Fan Zhang, Mohamed Elmeliegy, Thandranese Owens, Dr. Steven Zatechka, Daniel Groepper, and Dr. Vamshi Manda for their support and friendship throughout the project.

At last, I also would like to gratefully acknowledge the care and support of my husband and parents, who always encouraged me on the way along. 


\begin{abstract}
Increasing evidence suggests that inhibition of vascular endothelial growth factor (VEGF) can transiently normalize tumor vasculature, thereby improving delivery of systemic chemotherapy. Bevacizumab (BEV), an anti-VEGF antibody, has been shown to transiently normalize tumor vasculature by increasing tumor vessel maturity, decreasing tumor vessel permeability, and increasing tumor oxygenation in an Rh30 orthotopic rhabdomyosarcoma xenograft model. However, the effects of BEV on the pharmacokinetics of TPT and the antitumor activity of TPT have not been evaluated. This study aimed to investigate the effect of BEV on TPT systemic and tumoral pharmacokinetics and to determine how these changes affect the efficacy of TPT in the Rh30 mouse model.
\end{abstract}

Mice bearing Rh30 orthotopic xenografts were treated with BEV alone $(5 \mathrm{mg} / \mathrm{kg})$, TPT alone $(2 \mathrm{mg} / \mathrm{kg})$ or a combination of the two administered intravenously with different schedules. The pharmacokinetics of TPT, including TPT intratumoral penetration, as well as the efficacy of the monotherapy and combination therapy were evaluated. Population pharmacokinetic modeling and covariate analysis of TPT pharmacokinetics were performed using the maximal likelihood expectation maximization (MLEM) method in ADAPT 5 to predict the plasma and tumor concentration-time profile, to estimate the pharmacokinetic parameters of individual mouse and mice population, and to evaluate the effect of BEV on TPT systemic and tumoral pharmacokinetics. Tumor penetration was assessed by the tumor-to-plasma ratio of area under concentration-time curve (AUC). Tumor volume before and after the treatment were measured to evaluate the antitumor activity of the treatment regimen, and to assess the effect of BEV on the antitumor activity of TPT in Rh30 xenografts.

Covariate analysis showed a single dose of BEV was associated with the increased systemic elimination rate and clearance of TPT. Furthermore, a single dose BEV had a time-dependent effect on the tumor elimination rate of TPT. The elimination rate of TPT from tumor compartment increased when it was given 1 day after a single dose of BEV and gradually decreased to control level when TPT was given 3 days and 7 days after a single dose of BEV. Multiple doses of BEV had no effect on TPT pharmacokinetics. TPT penetration was not altered after administering multiple doses of $\mathrm{BEV}$, but a single dose of BEV produced a trend in changes of TPT penetration. Tumor efficacy was not dependent on the schedule of BEV and TPT. TPT significantly enhanced the antitumor activity of combination therapy while pre-treatment of BEV did not alter the antitumor activity of TPT. Tumor efficacy in MDBT groups was mainly due to the multiple doses of BEV and the antitumor activity of TPT was diminished.

The present work provides crucial insights into the effect of coadministration of BEV on the pharmacokinetic changes and antitumor activity of TPT. The increased TPT systemic elimination and clearance after single dose of BEV treatment may be due to the altered renal clearance by VEGF. The increased TPT elimination from tumor tissue after 1 day pre-treatment of BEV may be caused by a normalization of tumor vasculature. The 
overall effect of BEV on TPT pharmacokinetics as well as TPT penetration is determined by the net balance of the pharmacologic changes of tumor microenvironment by BEV. And the antitumor activity of combination is determined by the balance between angiogenesis inhibition-induced tumor cell starvation and the tumor cytotoxicity due to the exposure to cytotoxic drugs. This study highlights the complexity of pharmacokinetic $(\mathrm{PK})$ and pharmacodynamic (PD) interaction that may take place when antiangiogenic agent and cytotoxic drug are combined and cautions that more consideration and mechanistic investigation should be made before using a combination of anti-angiogenic agents with cytotoxic drugs for cancer treatment. 


\section{TABLE OF CONTENTS}

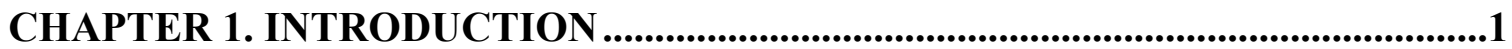

$1.1 \quad$ Drug Penetration in Solid Tumors ................................................................

1.1.1 Features of tumor microenvironment ...................................................... 1

1.1.2 Determinants for drug penetration in solid tumors.......................................2

1.1.3 Strategies to improve drug penetration in solid tumors..............................4

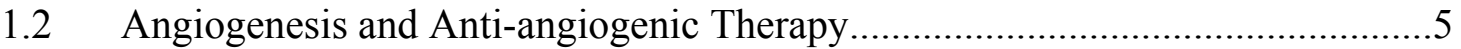

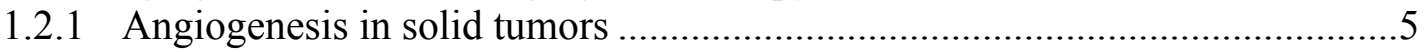

1.2.2 Role of vascular endothelial growth factor in tumor growth and

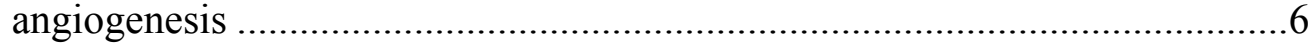

1.2.3 Anti-angiogenesis therapy and tumor vasculature normalization ..................6

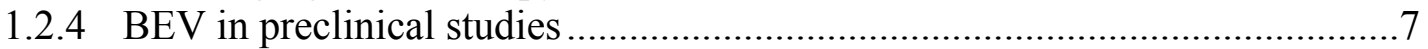

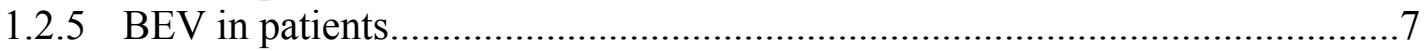

1.3 Rh30 Rhabdomyosarcoma as a Tumor Model ........................................... 9

1.3.1 Rhabdomyosarcoma .........................................................................

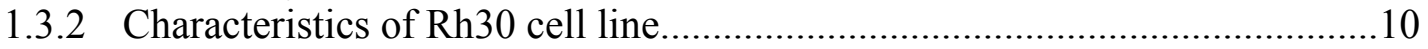

1.4 Methods to Evaluate Drug Penetration in Solid Tumors ..................................11

1.4.1 Homogenization and quantitative imaging................................................... 11

1.4.2 Microdialysis ................................................................................. 12

1.5 The Effects of Anti-angiogenic Agents on the Pharmacokinetics of

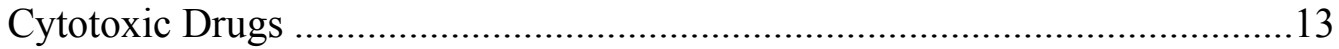

1.5.1 The effects of anti-angiogenic agents on cytotoxic drugs disposition in solid tumors .....................................................................................13

1.5.2 The effects of BEV on cytotoxic drugs disposition in solid tumors...............16

1.6 Pharmacokinetic Models of TPT in Preclinical Studies .................................17

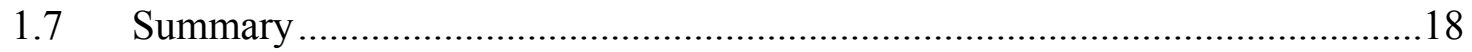

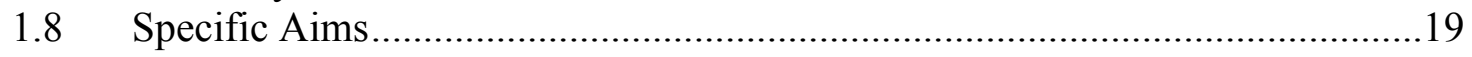

\section{CHAPTER 2. THE EFFECT OF BEVACIZUMAB ON THE \\ PHARMACOKINETICS OF TOPOTECAN IN A RH30 \\ RHABDOMYOSARCOMA XENOGRAFT.} .20

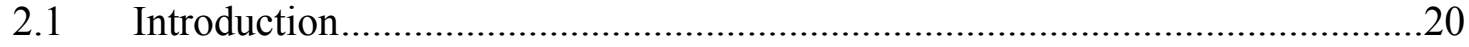

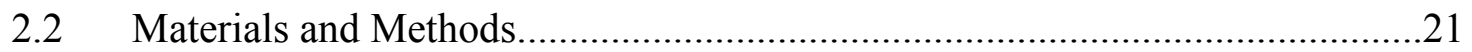

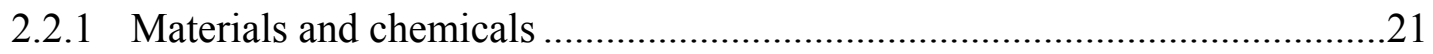

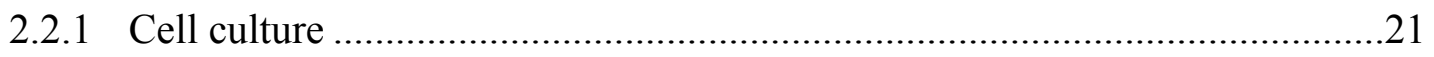

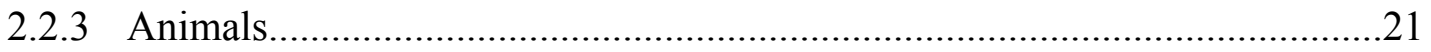

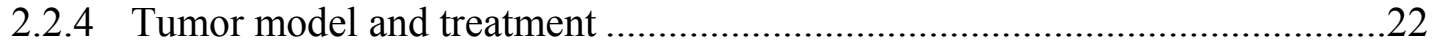

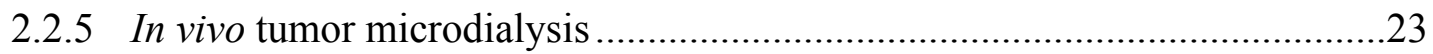

2.2.6 High-performance liquid chromatography analysis for PK studies ..............27

2.2.7 Pharmacokinetic model evaluation.............................................................27

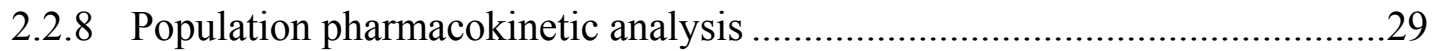

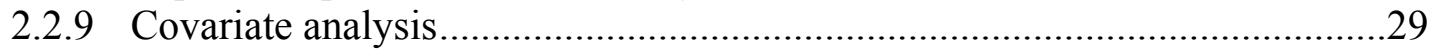

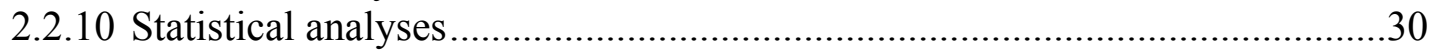

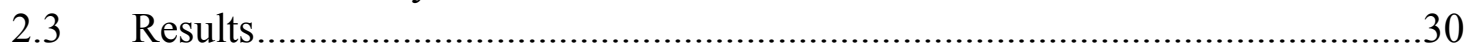


2.3.1 The effect of BEV on the pharmacokinetics of TPT

2.3.2 The antitumor activity of the combination therapy

CHAPTER 3. DISCUSSION AND CONCLUSIONS .........................................43

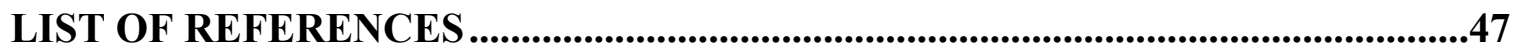

VITA 


\section{LIST OF TABLES}

Table 1. TPT penetration study design after a single dose of BEV treatment (SDBT)

Table 2. TPT penetration study design after multiple doses of BEV treatment (MDBT)

Table 3. TPT penetration after SDBT in mice bearing Rh30 RMS xenograft............33

Table 4. TPT penetration after MDBT in mice bearing Rh30 RMS xenograft ..........35

Table 5. Population PK parameters of TPT estimated after SDBT ..........................37

Table 6. Population PK parameters of TPT estimated after MDBT ..........................38 


\section{LIST OF FIGURES}

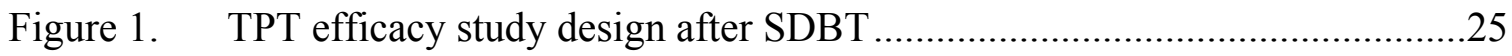

Figure 2. TPT penetration plus efficacy study design after MDBT ..............................26

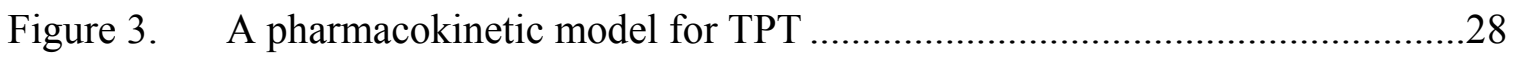

Figure 4. The modified multi-compartmental PK model for TPT ……........................28

Figure 5. Representative plasma and tumor disposition of TPT with/without SDBT in mice bearing Rh30 RMS xenograft .............................................31

Figure 6. Representative plasma and tumor disposition of TPT with/without MDBT in mice bearing Rh30 RMS xenograft............................................32

Figure 7. The effect of BEV on the intratumoral exposure, plasma exposure and intratumoral penetration of TPT after SDBT in mice bearing orthotopic Rh30 RMS xenograft

Figure 8. The effect of BEV on the intratumoral exposure, plasma exposure and intratumoral penetration of TPT after MDBT in mice bearing orthotopic Rh30 RMS xenograft .............................................................................36

Figure 9. The effect of treatment regimen on Ke and Kte ..........................................39

Figure 10. The effect of different schedule of SDBT combined with TPT on the growth of orthotopic Rh30 tumors

Figure 11. The effect of different schedule of MDBT combined with TPT on the growth of orthotopic Rh30 tumors 


\section{LIST OF ABBREVIATIONS}

\begin{tabular}{ll} 
ABC & ATP-binding cassette \\
ADA & Anti-drug antibodies \\
ARMS & Alveolar rhabdomyosarcoma \\
AUC & Area under concentration-time \\
AUCt & Area under concentration-time in tumor \\
AUCp & Area under concentration-time in plasma \\
BCRP & Breast cancer resistance protein \\
BEV & Bevacizumab \\
bFGF & Basic fibroblast growth factor \\
COG & Children's oncology group \\
CGH & Comparative genomic hybridization \\
CPT-11 & Irinotecan \\
CSF & Cerebrospinal fluid \\
CYP & Cytochrome p450 \\
ECF & Extracellular fluid \\
ECM & Extracellular matrix \\
ERMS & Embryonal rhabdomyosarcoma \\
HPLC & High-performance liquid chromatography \\
IFP & Interstitial fluid pressure \\
IIV & Inter-individual variability \\
IRS & Intergroup rhabdomyosarcoma study group \\
IC & Intracerebral \\
IP & Intraperitoneally \\
IV & Intravenously \\
MDBT & Multiple doses of bevacizumab treatment \\
MLEM & Maximal likelihood expectation maximization \\
MMPs & Matrix metalloproteinases \\
MMP-2 & Matrix metalloproteinase-2 \\
MT1-MMP & Membrane type 1 metalloproteinase \\
MVD & Microvessel density \\
NMR & Nuclear magnetic resonance \\
PBS & Phosphate buffered saline \\
PD & Pharmacodynamic \\
PDGF & Platelet-derived growth factor \\
PET & Positron emission tomography \\
PK & Pharmacokinetics \\
PTX & Paclitaxel \\
P-gp & P-glycoprotein 1 \\
QAR & Quantitative autoradiography \\
RD & Embryonal rhabdomyosarcoma cell line \\
RH30 & Alveolar rhabdomyosarcoma cell line \\
RMS & Rhabdomyosarcoma \\
SD & Standard deviation \\
& \\
\hline
\end{tabular}




$\begin{array}{ll}\text { SDBT } & \text { Single dose of bevacizumab treatment } \\ \text { SC } & \text { Subcutaneous } \\ \text { TIMP-2 } & \text { TIMP metallopeptidase inhibitor } 2 \\ \text { TMZ } & \text { Temozolomide } \\ \text { TNP-470 } & \text { O-(N-chloroacetyl-carbamoyl)-fumagillol } \\ \text { TPT } & \text { Topotecan } \\ \text { VEGF } & \text { Vascular endothelial growth factor } \\ \text { VEGFR } & \text { Vascular endothelial growth factor receptor }\end{array}$




\section{CHAPTER 1. INTRODUCTION}

\subsection{Drug Penetration in Solid Tumors}

\subsubsection{Features of tumor microenvironment}

Solid tumors are structurally heterogeneous and complex. They are composed of tumor cells and stromal cells such as endothelial cells, peri-vascular cells, fibroblasts and myofibroblasts that are embedded in the extracellular matrix and nourished by the vascular network [1]. Solid tumors have a unique microenvironment with several characteristics that distinguish them from the corresponding normal tissue. Abnormal solid tumor microenvironments are thought to be created by the interaction between the tumor vasculature and the cells within the tumor [2]. The three major recognized microenvironmental hallmarks of solid tumors are the abnormal vasculature, the compacted extra-vascular compartment, and the unfavorable metabolic environment [3].

The first hallmark of solid tumors-the abnormal vasculature-is composed of aberrant tumor angiogenesis, tortuous vascular architecture, heterogeneous vascular permeability, and irregular blood flow [4-6]. Angiogenesis is the physiological process of new capillaries generated from pre-existing blood vessels [7]. In normal tissue, angiogenesis is controlled by a precise balance between pro- and anti-angiogenic factors [8]. Blood vasculature in normal tissue consists of arterioles, capillaries and venules with distinct features and is characterized by dichotomous branching [2]. However, in pathological situations such as cancer, tumor cells can tilt the balance toward stimulatory angiogenic factors to drive vascular growth in order to grow and metastasize to other organs [9]. As a result, the tumor vasculature turns out immature and tenuous in nature. Furthermore, tumor vessels share all features of three types of vessels-arterioles, capillaries, and venules [2]. Thus tumor vasculature is marked by excessive branching loops and arteriolar-venous shunts [10]. In addition, the walls of tumor vessels are heterogeneous, with aberrant basement membranes, peri-vascular smooth muscle or pericytes in different regions [11]. Also, tumor cells can incorporate into vessel walls [12]. Thus tumor vessels are dilated, tortuous, disorganized, and have high permeability. However, although the overall permeability is higher in tumor vessels compared to normal blood vessels, some regions of tumor vessel walls can be normal or even thicker than normal blood vessel walls and have less permeability $[13,14]$. Moreover, blood flow is controlled by arterio-venous pressure and vasculature geometric resistance [1]. In solid tumors, decreased arterio-venous pressure, increased vasculature geometric resistance, and the compression of blood vessels by tumor cells reduces the overall blood flow and impair blood supply to the tumor cells [15-17]. In addition, the abnormality of vascular architecture, blood vessel wall and blood flow can vary with location, with time, and even in the same tumor region [18].

The second hallmark of solid tumors-the compacted extra-vascular compartment - is mainly displayed by the dysfunctional lymphatic system, interstitial 
hypertension and the pathologic extracellular matrix [19-21]. The major role of lymphatic vessels is to drain the interstitial fluid from peripheral tissue to blood vessels and to maintain the interstitial fluid balance in the tissue [19]. The rapid proliferation of tumor cells compresses blood vessels and lymphatic vessels [17]. Accordingly, the lymphatic system inside the solid tumors becomes dysfunctional and the blood vessels become structurally and functionally abnormal [4-6, 11, 14-18]. However, there are functional lymphatic vessels in the margin of the solid tumors or in the peri-tumor tissues [1]. Thus, the interstitial fluid is confined within solid tumors, and interstitial fluid pressure is uniformly high throughout the core of a solid tumor, while dropping dramatically in the tumor margin $[22,23]$. The extracellular matrix (ECM) consists of basement membrane and interstitial stroma. The basement membrane mainly contains collagen IV, laminin and proteoglycans, while the interstitial stroma mainly contains fibrillar collagens, fibronectin, hyaluronic acid, and fibril-associated proteoglycans [24]. In solid tumors, the components in ECM are dynamically changed and large amount of these components are overexpressed, attributable in part to the extensive synthesis of ECM [25-27].

Furthermore, the fast growth of tumor cells within a limited space also squeezes or compresses the ECM into a compacted pattern [28, 29].

The third hallmark of solid tumors-the unfavorable solid tumor metabolic environment-is the low levels of oxygen and acidic $\mathrm{pH}$ [30-32]. Due to an imbalance between tumor cell proliferation and vasculature development, multiple regions of cells in solid tumors are distant, such as $180 \mu \mathrm{m}$ away from blood vessels, so that oxygen cannot be transported and diffused to those regions $[33,34]$. This chronic effect of hypoxia results in multiple necrotic regions in solid tumors. Even though the tumor cells are close to the blood vessels, they can also undergo acute hypoxia due to the intermittent and irregular blood flow [35]. The low extracellular $\mathrm{pH}$ is due to increased production and reduced removal of $\mathrm{H}^{+}$ions [1]. The main sources of increased $\mathrm{H}^{+}$ions production are from anaerobic glycolysis under the hypoxia condition and from $\mathrm{CO}_{2}$ and $\mathrm{H}_{2} \mathrm{O}$ by carbonic anhydrase [36]. The reduced removal of $\mathrm{H}^{+}$ions is caused by abnormal microcirculation.

In conclusion, the microenvironment in solid tumors displays an aberrant vascular compartment, a compacted extra-vascular compartment, and unfavorable metabolic environment.

\subsubsection{Determinants for drug penetration in solid tumors}

After reaching the systemic circulation, therapeutic drugs quickly diffuse through the vasculature and distribute within the solid tumors. Drug penetration in solid tumors depends on several factors, including the physicochemical properties of the drug, formulation, and the delivery system of the drug as well as the neoplastic tissue microenvironment. For a formulated drug product, there are three major determinants or barriers in solid tumors that inhibit drug penetration from systemic blood circulation to the therapeutic target cells: the aberrant blood vessel architecture, the heterogeneous vessels' wall, and the compacted extracellular matrix [37]. 
The therapeutic agents depend on blood circulation to reach the targeted tissue, so the pathologic vasculature is the first determinant for drug penetration in solid tumors [38]. The distribution of the drug in tumor is governed by the blood vessel morphology and the blood flow rate in the tumor [39]. As discussed above, the number, length, diameter and geometric arrangement of blood vessels are irregular and the blood flow rate is fluctuated in solid tumors. Consequently, some regions are well perfused while other regions are totally unperfused within the same tumor [40]. Accordingly, the drug can accumulate in one region but not access another region at all. Furthermore, cessation of blood flow will reduce net tumor cell 'exposure' to the therapeutic agents in blood circulation and even intermittent decreases in blood flow will impact on the net distribution (AUC) of systemically administered agents [41].

Additionally, the blood vessel wall in solid tumors also affects the ability of therapeutic drugs to diffuse universally to extra-vascular space [42]. The blood vessel wall is heterogeneous and hyperpermeable with thick or thin basement membrane, less or more pericytes and maximum diameter of the irregular pores [12]. The leaky wall of the blood vessel is likely to yield higher drug uptake; however, interstitial hypertension prevents the convective transport of drug between intra-vascular and extra-vascular spaces [43]. Since the interstitial pressure is high throughout the core of the solid tumor but drops dramatically in the tumor margin, the drug tends to distribute in the tumor peripheral region rather than the center of it [23], resulting less drug penetration in the core of solid tumors.

Lastly, the condensed interstitium and unfavorable metabolic environment dramatically hinder the drugs from accessing tumor cells [25]. Tumor cells are highly packed, and the extracellular matrix is compressed by tumor cells, both of which reduce drug penetration in solid tumors [28]. Drug penetration and the efficacy of anticancer drugs are considerably decreased in tightly packed cells compared to loosely packed cells [44]. Similarly, the well organized and extensively interconnected collagen network in the extracellular matrix severely affects drug movement in the interstitium [25]. The harsh microenvironment with hypoxia and acidic $\mathrm{pH}$ may also impair drug penetration in solid tumors. For example, too much hypoxia leads the hypoxia-activated prodrugs (such as tirapazamine, AQ4N, and PR-104) to extensive metabolism, leaving no drug remaining to penetrate to the targeting sites [45]. And weak basic drugs (such as doxorubicin and mitoxantrone) or weak acidic drugs (such as methotrexate) have shown slower drug penetration in acidic solid tissues [46, 47].

Thus, there are multiple determinants in solid tumors that hinder a drug from effectively penetrating to the tumor cells and exerting its cytotoxic action. In order to overcome this obstacle, many investigators have developed useful strategies to improve drug penetration in solid tumors. 


\subsubsection{Strategies to improve drug penetration in solid tumors}

The strategies to improve drug penetration in solid tumors mainly fall into two categories: 1 . Normalize the tumor microenvironment including tumor vasculature and extracellular matrix (ECM). 2. Develop more effective delivery methods for drug penetration or modifying drug property for deeper penetration.

The first strategy that investigators tried is to ameliorate the microenvironment of solid tumors for drug penetration, normalizing the tumor vasculature and tumor matrix [42]. Overexpression of proangiogenic molecules such as vascular endothelial growth factor (VEGF), basic fibroblast growth factor (bFGF) and platelet-derived growth factor (PDGF) may be the major cause of the structurally and functionally abnormal vasculature in solid tumors [14]. Blocking these pro-angiogenic factors leads to increased apoptosis of endothelial cells and therefore elimination of immature blood vessels, creating tumor vessels with closer resemblance to normal vessels in structure and function [14]. The features of tumor vasculature normalization may include the reduction of microvessel density, interstitial fluid pressure (IFP), and hypoxia as well as the increase of blood flow and perfusion rate [48]. Numerous studies have shown that normalization of tumor vasculature via anti-angiogenesis therapy improves cytotoxic drug penetration in solid tumors. Increased paclitaxel (PTX) concentration in two solid tumor tissues after combining with BEV, an anti-VEGF monoclonal antibody, has recently been observed accompanying the downregulation of vascular permeability [49]. In a MX-1 human breast cancer xenograft, the intratumoral PTX concentration in mice treated with a single dose of PTX $30 \mathrm{mg} / \mathrm{kg}$ plus a single dose of BEV $5.0 \mathrm{mg} / \mathrm{kg}$ was significantly higher than in the tumor treated with PTX $30 \mathrm{mg} / \mathrm{kg}$ alone $(5.75 \pm 0.31 \mu \mathrm{g} / \mathrm{g}$ vs. $4.00 \pm 0.85 \mu \mathrm{g} / \mathrm{g})$. And the PTX concentration in tumor treated with PTX $30 \mathrm{mg} / \mathrm{kg}$ plus BEV $5.0 \mathrm{mg} / \mathrm{kg}$ was equivalent to that in the tumor treated with either 60 or $100 \mathrm{mg} / \mathrm{kg}$ of PTX alone. An increase in PTX concentration by BEV was also observed in an A549 human lung cancer xenograft model. In the same MX-1 model, vascular permeability in the tumor was significantly decreased by treatment with BEV. DC101, a VEGF-receptor-2 antibody, has been shown to normalize tumor vasculature and increase BSA penetration in several solid tumors [50]. Combining sunitinib, an inhibitor of several tyrosine kinase receptors (including vascular endothelial growth factor receptors, platelet-derived growth factor receptors and stem cell factor receptor), with temozolomide significantly increases the penetration of temozolomide in human glioma xenografts. This enhanced penetration is associated with an improved "vascular normalization index" incorporating the microvessel density (MVD) and protein expression of $\alpha$-SMA and collagen IV [51]. Other than the normalization of vasculature, the normalization or degradation of ECM also can improve the uptake and penetration of drugs. As discussed above, the well organized collagen network slows the penetration rate of cytotoxic drugs. The addition of collagenase induces a two-fold increase in tumor uptake and improves the distribution of the monoclonal antibody TP-3 in a human osteosarcoma xenograft by degrading ECM while decreasing IFP and microvascular pressure [52]. Furthermore, reducing the packing density of tumor cells by chemotherapy itself can relieve the compacted ECM by killing tumor cells, decompressing blood vessels and decreasing IFP, which results in increased 
drug penetration. Pre-treatment with $1 \mu \mathrm{M}$ non-radiolabeled paclitaxel enhanced the penetration rate of $\left[{ }^{3} \mathrm{H}\right]$ paclitaxel in a human pharynx FaDu xenograft [53].

The second strategy that researchers have used is a targeting delivery system or improved drug property to increase the drug distribution in solid tumors. The targeting delivery system mainly uses two principles: passive targeting or active targeting [54]. Passive targeting involves enhancing the drug carrier permeability and retention in solid tumors by designing the particle size too large to diffuse through normal blood vessels but small enough to exit the abnormally large pores on the tumor vessel walls to extra-vascular space. With more drugs in the extra-vascular space by the passive targeting system, relatively more drug will diffuse into solid tumors and restrict it in the tumor tissue due to lack of a functional lymphatic system [55]. The active targeting strategy employs the specific tumor microenvironment by attaching ligands to the carrier surface that can bind to tumor specific receptors and antigens through molecular recognition. These receptors and antigens include the cancer cell surface antibodies [56], folate receptor [57], transferrin receptors [58] and angiogenic vascular surface proteins [59]. Another modification for deeper drug penetration is to use live bacterial vectors such as Salmonella sp, Clostridium sp and Escherichia coli based on their tumor-colonizing characteristics [60]

In summary, numerous strategies have been developed to increase cytotoxic drug penetration in solid tumors. The normalization of tumor vasculature is one of the most popular methods to enhance drug penetration.

\subsection{Angiogenesis and Anti-angiogenic Therapy}

\subsubsection{Angiogenesis in solid tumors}

Angiogenesis is the physiological process of new capillaries generation from pre-existing blood vessels [7]. In normal tissue, angiogenesis is precisely regulated by keeping a balance between pro-angiogenic factors, such as VEGF and PDGF, and anti-angiogenic factors, such as thrombospondin-1 and angiostatin [61]. Regulated angiogenesis provides organs and tissue sufficient nutrition and oxygen to grow and recover from wound healing [62]. Under pathological conditions, the balance of pro-angiogenic factors and anti-angiogenic factors is broken and leads to dysfunctional and structurally abnormal vasculature. Unregulated angiogenesis plays a critical role in solid tumor growth and metastasis [63]. As in normal tissue, a tumor beyond $0.5 \mathrm{~mm}$ in diameter relies on angiogenesis to supply nutrition and oxygen for survival and growth [64]. Furthermore, the dilated and leaking intra-tumoral blood vessels allow the tumor cells to enter the blood circulation and metastasize to distant organs [65]. In this context, the anti-angiogenesis approach as a tumor treatment strategy has been investigated extensively. 


\subsubsection{Role of vascular endothelial growth factor in tumor growth and angiogenesis}

Vascular endothelial growth factor-A (also termed VEGF) is a key angiogenic mediator [65]. As early as 1996, the group at Genentech led by Dr. Napoleone Ferrara showed that even loss of a single $V E G F$ allele resulted in several impaired developmental vasculature abnormities and was lethal in the mouse embryo between days 11 and 12 [66]. Furthermore, the overexpression of VEGF is frequently found in human solid tumors $[67,68]$. A elevated expression of VEGF is found to correlate to tumor angiogenesis, progression and survival in patients [69].

There are multiple isoforms of VEGF, ranging from 121 to 206 amino acids, and all the isoforms can bind to two receptors: VEGFR-1 and VEGFR-2 [70]. The major activities of VEGF in endothelial cells are regulated by signaling via VEGFR-2 [71]. Blockage of VEGF/VEGFR pathways is sufficient to suppress vasculature in numerous solid tumor models and inhibition of these pathways also results in tumor growth suppression [72]. Among these VEGF/VEGFR inhibitors, BEV, a monoclonal antibody specific for VEGF, is the most extensively studied candidate in preclinical and clinical settings.

\subsubsection{Anti-angiogenesis therapy and tumor vasculature normalization}

In 1971, Dr. Folkman [73] proposed that the inhibition of angiogenesis might be an effective strategy to treat human cancer. Thereafter, a large body of literature discussed the investigation of angiogenesis inhibitors. Driven by this hypothesis, Dr. Folkman's lab developed the first anti-angiogenic agents in the early 1980s [74, 75], and additional synthetic and endogenous angiogenesis inhibitors were discovered by numerous labs [76-79]. In the mid-1990s, the angiogenesis inhibitors started to enter clinical trials. In 2004, the first anti-angiogenic agent, bevacizumab (BEV), was approved by the FDA for colorectal cancer [80]. Currently, over 2,000 clinical trials in USA are testing drugs that have varying degrees of anti-angiogenic activity for different types of cancers; over 300 of these clinical trials are in phase III.

After decades of effort, the tumor normalization concept emerged in 2005 from Dr. Jain's research [14]. The concept is: under a pathological condition, the imbalance of the pro-angiogenic factors and anti-angiogenic factors persists. Restoring this imbalance or making the imbalance favor the anti-angiogenic factors may lead to vascular regression or a close to normal condition and ultimately lead to the prevention of tumor growth and metastasis. Additionally, Dr. Jain proposed a vascular normalization window when using proper dosing and scheduling of angiogenesis inhibitors. This concept was widely accepted by the field and, indeed, numerous investigators identified this normalization window. For instance, the recent study, in which mice bearing orthotopic U87 xenografts were treated with BEV or interferon $\beta$, showed that the anti-angiogenic agent treatment induced significant changes in tumor vascular physiology, improving intra-tumoral oxygenation and enhancing the antitumor activity of ionizing radiation [81]. 
However, the window for human U87 tumor model is transient and only lasts about 5 days.

\subsubsection{BEV in preclinical studies}

Numerous studies have been conducted to investigate the pharmacological effect of BEV in solid tumor xenograft models. Investigators have mainly focused on tumor growth inhibition via disruption of tumor vasculature and the inhibition of tumor metastasis.

In general, studies of the anti-VEGF effect usually employed vascular changes and tumor growth inhibition as primary end points and have showed that BEV significantly decreased vessel density and tumor growth regardless of tumor types. Dr. Finn et al. showed that administration of BEV $5 \mathrm{mg} / \mathrm{kg}$ intra-peritoneal twice a week significantly decreased microvessel density, decreased human serum alpha-fetoprotein and delayed tumor progression for treated mice compared to the controls in a human hepatocellular orthotopic xenograft model [82]. Dr. Okada et al. administered BEV 2 $\mathrm{mg} / \mathrm{kg}$ intra-peritoneal twice a week for 8 weeks and found that BEV significantly inhibited tumor growth by $48 \%$ in volume at the end of the experiment [83]. Meanwhile, the intratumoral microvessel density was significantly decreased in the BEV treatment group compared to the control group and a positive correlation was found between tumor volume and microvessel density.

VEGF plays a critical role in the tumor angiogenesis and angiogenesis is a feature of growth and invasion in primary neoplasms and their metastases. Furthermore, BEV has been shown to control metastatic colorectal carcinoma, metastatic breast carcinoma, and metastatic non-small cell lung carcinoma [84-86]. However, there are also several case reports about more cancer metastasis after BEV treatment [87, 88]. Thus some investigators start to assess the anti-VEGF effect on tumor metastasis. Imaizumi et al. used a peritoneal metastasis model to determine the effect of BEV on peritoneal dissemination from gastric cancer and showed BEV had a significant effect on peritoneal dissemination suppression [89]. More directly, Dr. Yang et al. examined the effect of $\mathrm{BEV}$ on tumor cell survival, invasiveness in vitro and metastasis in vivo [90]. The results showed that BEV decreased in vitro growth and invasion and further suppressed in vivo hepatic metastasis of ocular melanoma cells.

Based on the promising tumor vasculature and growth inhibitory effects of BEV in preclinical models, the application of BEV was moved forward to clinical trials.

\subsubsection{BEV in patients}

Although BEV showed a significant effect on tumor vasculature changes, tumor growth, metastasis and survival rate in preclinical studies, BEV alone only provides modest survival benefits in patients [91]. However, it showed that anti-VEGF treatment 
enhanced the tumor response to conventional chemotherapy. Thus, currently the primary use of BEV in clinical patients is in combination with conventional chemotherapy [92].

The precise mechanism of how BEV enhances the conventional regimens is unclear, but at least four mechanisms have been proposed:

1. Enhancement effect of conventional cytotoxic drug penetration by tumor vasculature normalization [14].

2. Synergic effect of BEV and cytotoxic drugs on tumor cells that express and depend on VEGF for growth and survival [93].

3. The anti-angiogenic effect of cytotoxic drugs facilitates the VEGF depletion by BEV [94].

4. The additive effect of BEV and cytotoxic drugs on both tumor cells and endothelial cells [95].

The overall improved outcome due to BEV, combined with conventional cytotoxic drugs, is the consequence of one or more of the mechanisms mentioned above functioning together. Clinical studies have showed that the addition of BEV to conventional chemotherapy provides significant survival benefits on cancer patients with diverse solid tumor types such as breast cancer [96], colorectal cancer [80, 97, 98] and non-small-cell lung cancer (NSCLC) [99-101]. Dr. Miller et al. compared the progression-free survival (PFS) and overall survival (OS) for patients with metastatic breast cancer receiving PTX alone and PTX plus BEV [96]. This phase III trial indicated that PTX plus BEV significantly prolonged PFS as compared with PTX alone (median, 11.8 vs. 5.9 months; hazard ratio for progression, $0.60 ; p<0.001)$ and increased the objective response rate $(36.9 \%$ vs. $21.2 \%, p<0.001)$. However, the OS was similar in the two groups (median, 26.7 vs. 25.2 months; hazard ratio, $0.88 ; p=0.16$ ). Dr. Hurwitz et al. compared the PFS and OS for patients with metastatic colorectal cancer receiving irinotecan, bolus fluorouracil and leucovorin (IFL) plus BEV and IFL plus placebo [80]. The results showed that IFL plus BEV significantly prolonged OS as compared to ILF plus placebo (median, 20.3 vs. 15.6 months; hazard ratio, $0.66 ; p<0.001$ ). Also, IFL plus $\mathrm{BEV}$ significantly prolonged PFS when compared to IFL plus placebo (median, $10.6 \mathrm{vs}$. 6.2 months; hazard ratio, $0.54 ; p<0.001$ ) and the corresponding rates of response were $44.8 \%$ and $34.8 \%(p=0.004)$. Dr. Johnson et al. assessed the benefit of addition of BEV to paclitaxel and carboplatin (PC) in patients with previously untreated locally advanced or metastatic NSCLC [101]. Compared with the control arm, treatment with PC plus $\mathrm{BEV}$ resulted in a higher response rate $(31.5 \%$ vs. $18.8 \%)$, longer median time to PFS (7.4 vs. 4.2 months) and a modest increase in OS (17.7 vs. 14.9 months). Among these tumor types, rhabdomyosarcoma is one which needs to be characterized and elucidated further for the use of BEV in addition to conventional chemotherapy, especially the alveolar subtype. 


\subsection{Rh30 Rhabdomyosarcoma as a Tumor Model}

\subsubsection{Rhabdomyosarcoma}

Rhabdomyosarcoma (RMS) arises from immature cells that are developed to form striated skeletal muscle, but it can virtually arise at any site and in any tissue in the body except bone [102]. Head and neck are the most common regions where RMS occurs (approximately 40\%); the genitourinary sites and the extremities are the next most frequent regions where RMS arises (approximately 20\% each); and the remaining 20\% RMS initiates from other sites, such as parameningeal sites and the retroperitoneum [103].

The two most common pathologic types of RMS are embryonal and alveolar[102]. Embryonal rhabdomyosarcoma (ERMS) is the most common RMS seen in infants and young children, comprising of more than $60 \%$ of all RMS. The ERMS cells [104] exhibit the normal developing muscle cells, such as stellate undifferentiated mesenchymal cells, elongated myoblasts, multinucleated myotubes, and fully differentiated myofibers. ERMS has a better prognostic outcome [105-107] than other subtypes and tends to occur in the genitourinary tract, head and neck, and abdomen. About $20 \%$ of all rhabdomyosarcoma are of the alveolar histology (ARMS) [108], in which cells look like the mature muscle cells in a 10-week-old fetus. ARMS grows much faster than ERMS, needs more intensive treatment, has a higher relapse incidence and worse prognostic outcome[109].

RMS occurs more frequently in children and adolescents ( $<20$ years) than in adults [110]. It is one of the typical tumors of childhood and adolescence, accounting for more than $50 \%$ of soft tissue sarcomas in these age groups. Its annual incidence is 4.3 cases per million people younger than 20 years [103]. The incidence of ERMS is much higher in the age group 0-4 compared to ARMS, while the incidence of ARMS is fairly stable throughout age group 0-19 [111].

The current treatment for RMS [103] requires a multidisciplinary approach by the pediatric oncologist, medical oncologist, surgeon, pathologist, and radiologist, and multimodality care: surgical resection combined with radiation therapy, followed by chemotherapy. The treatment is based on careful pre-treatment staging and grouping $[102,103]$ as suggested by the intergroup rhabdomyosarcoma study group (IRS) and now named the soft tissue sarcoma committee of the children's oncology group (COG).

The 5-year overall survival rate of children and adolescents with RMS is significantly higher than adult patients [110] (61\% $\pm 1.4 \%$ and $27 \% \pm 1.4 \%$, respectively). However, $30 \%$ of young patients experience recurrence, and $50 \%$ to $95 \%$ of these patients die of progressive disease [112]. The survival rate significantly differs for different RMS subtypes and depends on the tumor sites. The 5-year survival rate of patients with ERMS or RMS in surgically favorable sites is much higher compared to patients with ARMS or those with primary sites in the parameningeal and other surgically 
non-favorable sites [109]. So developing new agents or new strategies to increase the current agent efficacy is urgently needed for the treatment of RMS, especially ARMS.

\subsubsection{Characteristics of $\mathrm{Rh} 30$ cell line}

The Rh30 cell line we chose for the tumor xenograft model is highly complex in genetic profile and overexpression of VEGF, VEGFR and MMP-2, which is associated with more aggressive invasion behavior and is characterized as an ARMS subtype.

ARMS is genetically characterized by reciprocal translocations that generate the fusion gene $P A X 3-F O X O 1 A$ (PF) or $P A X 7-F O X O 1 A$. The Rh30 cell line is characterized as ARMS cell line and positive for PAX3-FOXO1A fusion gene. By applying any of several techniques such as spectral karyotyping, fluorescence in situ hybridization, comparative genomic hybridization $(\mathrm{CGH})$, and microarray $\mathrm{CGH}$, Rodriguez-Perales et al. [113] found Rh30 had a wide range of chromosome numbers, more than 50 chromosome rearrangements, amplification of the hybrid gene, 24 DNA changes and 21 gene copy changes. p53 is also mutated in the Rh30 cell line with a codon 280 A to T transversion (arginine to serine) [114].

VEGF is overexpressed in the Rh30 cell line, which provides a rationale for our selection of it for anti-VEGF therapy. Moreover, the overexpression of MMP-2 renders Rh30 more invasive than ERMS cell lines [115]. Onisto et al. studied the MMP-2, MT1-MMP, TIMP-2, VEGF and VEGF receptors in several ARMS, ERMS and undifferentiated cell lines and tried to correlate the metastatic potential with the matrix metalloproteinases (MMPs) and angiogenesis-related factors. The results showed that Rh30 had increased expression levels of both $\mathrm{VEGF}_{165}$ and $\mathrm{VEGF}_{121}$ isoforms as well as VEGFR-1 at mRNA and protein. Also the elevated expression of MMP-2 at mRNA and protein levels correlated with the invasive behavior in vitro.

Comparing the ERMS cell line RD, Rh30 cell line is more sensitive to camptothecin and its derivative TPT [116], and the effective schedules of exposure of Rh30 xenografts to TPT have been identified [117]. By high-throughput screening, Zeng et al. showed that camptothecin and TPT inhibited cell proliferation and induced cell apoptosis more effectively in Rh30 than in RD (ERMS) cells. Ectopic expression of the fusion protein $\mathrm{PF}$ in RD cells significantly increased their sensitivity to camptothecin and TPT, whereas siRNA knockdown of PF decreased the sensitivity of Rh30 cells to camptothecin and TPT [116]. Furthermore, Dr. Stewart and his collaborators showed that TPT was highly schedule-dependent in Rh30 cells and that only daily exposure of TPT achieved complete regressions of Rh30 xenografts [117]. The dose and schedule for complete regression in Rh30 xenografts was to give TPT daily $0.6 \mathrm{mg} / \mathrm{kg}$ for 2 weeks, repeated every 21 days for three cycles. However, preclinical and clinical studies showed that rhabdomyosarcoma is resistant to TPT $[118,119]$. To overcome the drug resistant and increase its efficacy, one of the approaches is to increase its intratumoral concentration. Thus, we used Rh30 orthotopic rhabdomyosarcoma xenograft as a tumor 
model to investigate the effect of schedule-dependent anti-VEGF treatment on TPT disposition and tumoral penetration in an orthotopic model of RMS in mice.

\subsection{Methods to Evaluate Drug Penetration in Solid Tumors}

\subsubsection{Homogenization and quantitative imaging}

Several techniques [120] have been used to quantitate drug concentration in solid tumors, including collecting tumor homogenate, using noninvasive quantitative imaging and sampling by microdialysis, which will be discussed in detail in the following section.

The use of tumor homogenates is a widely used technique to determine drug concentration in normal and tumoral tissues. This method was used frequently in early drug pharmacokinetic studies [121-123] and currently is still commonly employed in this field [124, 125]. Although this approach has multiple advantages (such as easy to implement, achieving results quickly, and getting large amount of samples for PK/PD studies simultaneously), it has several disadvantages as well. The concentration obtained by this sampling method is the total drug concentration, including protein bound and unbound drugs that are present in tumor vascular, the interstitial space, and intratumoral compartments. This mixed concentration complicates the interpretation of the results since the unbound free drug is the pharmacologically active drug. Additionally, the total drug from these compartments also limits insight into underlying drug distribution and transport mechanisms. Another drawback of this approach is that only one sample can be obtained from each animal. In order to get adequate information for the drug disposition in the tumor, one has to sacrifice multiple animals to get adequate data to interpret drug disposition or penetration, which requires considerable animal resources. Furthermore, this approach also leads to wide inter-animal variability, which can complicate the interpretation of the data.

An alternative way to quantify drug concentration in the tumor tissue is to use quantitative imaging such as positron emission tomography (PET) [126, 127], quantitative autoradiography (QAR) [128], and nuclear magnetic resonance (NMR) [129, 130]. PET scanning combines computerized tomography (CT) and radioisotope imaging. By injecting radiolabeled drug, it is possible to map drug distribution in the body or the target tissue-tumor. When combining pharmacokinetic tools, it is also possible to determine the kinetic changes of the radiolabeled drug and various physiological parameters [131]. While PET has low spatial resolution, QAR can measure radiolabeled drug concentration in small regions of the target tissue. However, the main limitation of these two techniques is the instability of isotope. Therefore, synthesis of the radioisotope must take place immediately before the in vivo experiment, and correction for the decay of the isotope is essential to obtain reliable results. Furthermore, both of these two techniques cannot distinguish between parent drugs and metabolites, nor between protein-bound drugs and free drugs [120]. While NMR is able to differentiate between parent drug and its metabolites as well as protein bound and unbound drugs, it has very 
low sensitivity. Additionally, it requires a long time to obtain signals while keeping small animal in a fixed position, which adversely affects the time resolution. Furthermore, the spatial resolution of NMR is also limited [132]. Nevertheless, noninvasive imaging techniques may reduce inter-individual variability since multiple time samples can be obtained from each animal [120].

\subsubsection{Microdialysis}

Currently, the microdialysis technique has acquired more attention than these techniques mentioned above and is widely accepted by many studying drug penetration to tissues and tumors. Microdialysis sampling has gained popularity [133] in preclinical and clinical pharmacokinetic studies since it was introduced by Delgado [134] in 1972 for its application in preclinical pharmacokinetic studies. This technique involves the insertion of a semi-permeable probe affixed to the inlet and outlet tubing. The probe virtually can be placed in any tissue such as brain, blood, liver, skin and solid tumors [135-139]. It is perfused by a solution that closely matched to the medium of the surrounding tissue, and the analyte is collected by the diffusion over the probe membrane and down its concentration gradient from the target tissue into the perfusate, which runs continuously out to the outlet tubing after drug administration.

Microdialysis has a number of advantages for in vivo sampling of drugs. The perfusion solution is isotonically matched to tissue extracellular fluid, so there is no fluid loss for the continuous sampling and minimal interruption to the integrity of PK determinants. Secondly, collecting serial samples from one animal not only reduces the number of animals needed but also minimizes the inter-individual variability of the data [120]. More importantly, the microdialysate contains only protein unbound free drug, which may facilitate a clear pharmacological interpretation and readily support physiologically based PK/PD models [140, 141]. Additionally, microdialysis is applicable to measure parent drug and its metabolites simultaneously since radiolabeled drugs are unnecessary $[142,143]$. Furthermore, microdialysis only samples molecules with smaller molecular weight than the probe molecular weight cut-off, such as 5 or 20 $\mathrm{kDa}$. So it automatically excludes a lot of drug degradation enzymes and cleans up the samples, thus avoiding the ex vivo enzymatic degradation and pre-analysis processing.

The main limitation of microdialysis is the probe calibration or in vivo recovery assessment to get the true concentration in the surrounding tissue that contradicts the advantage of the reduction of animal subjects and inter-individual variability. Secondly, since only a very small volume (15-50 microliters) of sample can be retrieved from each time point, it requires more sensitive analytical methods to quantify the drug concentration in the samples. Third, the invasiveness of the probe insertion also raises concerns of the interruption of the physiological system. However, investigators [144] showed that if the animals are given adequate time (e.g., 24 hours for intracranial microdialysis) to recover, the tissue damage can be managed. Despite these limitations, the ability to continuously sample unbound and presumably active drugs in the specific 
tissue region renders microdialysis a powerful and appealing technology that is applied more and more in drug delivery and pharmacokinetic studies.

\subsection{The Effects of Anti-angiogenic Agents on the Pharmacokinetics of Cytotoxic Drugs}

\subsubsection{The effects of anti-angiogenic agents on cytotoxic drugs disposition in solid tumors}

The effects of anti-angiogenic agents on cytotoxic drug disposition in solid tumors are paradoxical: Some investigators found that anti-angiogenic agents decrease the penetration of cytotoxic drugs into solid tumors; some indicated that anti-angiogenic agents increase the uptake of cytotoxic drugs into solid tumors, while others demonstrated that the delivery of cytotoxic drugs into solid tumors does not change after the administration of anti-angiogenic agents.

Several studies showed pre-treatment with angiogenesis inhibitors decreased temozolomide (TMZ) concentration or exposure in rat glioma xenografts. In 1996, Dr. Gallo et al. examined the TMZ interstitial fluid concentration by using the microdialysis technique in a subcutaneous (SC) rat C6 glioma xenograft model after multiple doses of the angiogenesis inhibitor O-(N-chloroacetyl-carbamoyl)-fumagillol(TNP-470) [145]. TNP-470 (30 mg/kg) was given SC on days $6,8,10,12$, and 14 following tumor implantation. On day 15, control (no TNP-470) and treated rats received $40 \mathrm{mg} / \mathrm{kg}$ of TMZ intra-arterially. Plasma and interstitial fluid samples were collected for $8 \mathrm{~h}$ and non-compartmental methods were used for pharmacokinetic modeling. The results of this study indicated that the mean TMZ area under the interstitial fluid concentration-time curve was reduced by $25 \%$ in the TNP-470-treated group compared to the control. In a subsequent study, the same group determined the tumor distribution of TMZ in SC and intracerebral (IC) rat glioma models with the overexpression of VEGF (V+) after anti-angiogenic agent TNP-470 [146] administration. TNP-470 (30 mg/kg) was administered to the animals SC every other day for total 5 doses (SC tumor model) or 4 to 6 doses (IC model) which is coincided with the presentation of the central nervous system symptom [146]. The day after the last dose of TNP-470, TMZ was administrated intra-arterially to achieve steady-state plasma concentration of $40 \mu \mathrm{g} / \mathrm{ml}$. The steady-state concentration of TMZ in tumor ECF and plasma as well as the concentration of TMZ metabolite 5-(3-methyltriazen-1-yl) imidazole-4-carboximide (MTIC) in tumor ECF were determined and microvessel density (MVD) was quantitated using an anti-CD31 method. In both the $\mathrm{V}+\mathrm{SC}$ and $\mathrm{V}+\mathrm{IC}$ models, significant reductions in TMZ tumor concentrations and tumor-to-plasma concentration ratios compared with control after TNP-470 treatment were observed, being reduced an average of $25 \%$ and $50 \%$ in the SC and IC tumors, respectively. MTIC concentrations in V+ SC tumors also were reduced by $50 \%$ after TNP-470 administration. Consistent with the reduction of TMZ and MTIC concentration in tumor ECF, MVD was reduced by TNP-470 compared with vehicle control in the V+ SC and V+ IC tumors. Additionally, Gallo et al. also determined the 
tumor distribution of TMZ in $\mathrm{V}+\mathrm{SC}$ and $\mathrm{V}+\mathrm{IC}$ rat glioma models after another anti-angiogenic agent 3-[(2,4-dimethylpyrrol-5-yl)methylidenyl] indolin-2-one (SU5416) administration [147]. SU5416 $(25 \mathrm{mg} / \mathrm{kg})$ or vehicle control was administrated intraperitoneally (IP) daily for a total of nine doses. Two days after the last dose of SU5416 or vehicle control, TMZ was administration as a steady-state infusion regimen to achieve plasma concentrations of $20 \mu \mathrm{g} / \mathrm{ml}$. In V+ SC tumors, a $24 \%$ reduction in steady-state plasma TMZ concentration as well as a $21 \%$ reduction in tumor-to-plasma concentration ratio was documented compared with controls. This reduction was also accompanied by a 20 to $35 \%$ reduction in MVD. In contrast with TNP-470 study, In a V+ IC tumor xenograft model, steady-state plasma TMZ concentration and tumor-to-plasma ratio were significantly increased by 2 -fold after SU4516 treatment compared to controls. The authors discussed that the differential effects of SU4516 in tumor distribution of $\mathrm{TMZ}$ in $\mathrm{V}+\mathrm{SC}$ and $\mathrm{V}+\mathrm{IC}$ tumor models may be attributed to the microdialysis sampling site, peripheral versus central, and the dimethyl sulfoxide administration vehicle.

In contrast to the reduction of cytotoxic drug penetration and concentration in tumor tissue after antiangiogenesis therapy, there were publications showed that antiangiogenic agents can improve the drug delivery to tumor site in addition to the observation in V+ IC rate model discussed in above paragraph. Gallo and colleagues published two papers $[51,148]$ that demonstrated that a lower dose of sunitinib significantly increased TMZ tumor distribution in two mice glioma xenografts when compared to a higher dose or control group. In mice bearing SF188V+ human glioma xenografts, vehicle, sunitinib $(10 \mathrm{mg} / \mathrm{kg}$ ) or sunitinib $(40 \mathrm{mg} / \mathrm{kg})$ was given everyday orally up to 14 days [51]. One day after the last dose of vehicle or sunitinib, TMZ was administrated as a single oral dose at $20 \mathrm{mg} / \mathrm{kg}$. Both sunitinib dosage $10 \mathrm{mg} / \mathrm{kg}$ and 40 $\mathrm{mg} / \mathrm{kg}$ increased temozolomide tumor distribution by using tumor-to-plasma AUC ratio compared to control group. However, only $10 \mathrm{mg} / \mathrm{kg}$ group reached statistical significance $(p<0.05)$. Furthermore, PD analysis indicated that the increased TMZ penetration was associated with an improved vascular normalization index (VNI). The VNI was proposed as an indication of the number of tumor vessels with relatively good quality by incorporating MVD, protein expression of $\alpha$-SMA and collagen IV. In a separate paper [148], Gallo et al. used IC U87MG as mice tumor model to investigate the effect of sunitinib on tumor distribution of TMZ. Vehicle, sunitinib $(20 \mathrm{mg} / \mathrm{kg})$ or sunitinib $(60 \mathrm{mg} / \mathrm{kg})$ was given everyday orally up to 7 days. One day after the last dose of vehicle or sunitinib, TMZ was administrated to achieve steady-state. The tumor-to-plasma steady-state TMZ concentration ratio was significantly increased in the $20 \mathrm{mg} / \mathrm{kg}$ sunitinib group compared to either the control or $60 \mathrm{mg} / \mathrm{kg}$ sunitinib groups. Again, the increased in the tumor penetration of TMZ was significantly correlated to the VNI. Also, Wildiers et al. observed a trend of increased intratumoral irinotecan (CPT-11) concentration in nude NMRI mice bearing a colon adenocarcinoma xenograft after 1 week pre-treatment of anti-VEGF mAb A4.6.1 [149]. $200 \mu \mathrm{g}$ A4.6.1 or placebo was administrated IP at day 0 and day 4 and CPT-11 was given IP on day 7 . After one hour of CPT-11 administration, plasma and tumor concentration was measured to assess the CPT-11 intratumoral penetration. In A4.6.1 treated mice, a trend to increased intratumoral CPT-11 concentration was observed ( $p=0.09$ ). PD analysis showed MVD was significantly decreased while tumor perfusion was significantly increased. In 
efficacy study, CPT-11 was equally effective with or without pretreatment with A4.6.1. The authors concluded that tumor vascular function and tumor uptake of anticancer drugs improved with VEGF-blocking therapy. Finally, as discussed in the second paragraph of section 1.1.3, increased PTX concentration in two solid tumor tissues after a single dose of BEV $(5 \mathrm{mg} / \mathrm{kg})$ has recently been observed accompanying the downregulation of vascular permeability [49]. Consistent with the increased intratumoral PTX concentration, the antitumor activity of BEV at $5 \mathrm{mg} / \mathrm{kg}$ in combination with PTX at 20 or $30 \mathrm{mg} / \mathrm{kg}$ was significantly higher than that of either agent alone. The authors concluded that the synergistic antitumor activity of PTX and BEV in combination may be a result of the increase in PTX concentration in tumor resulting from the downregulation of vascular permeability when co-administered with BEV.

The underlying reasons for this paradoxical phenomenon have been discussed by numerous investigators. As mentioned in section 1.1, the poorly organized and irregular tumor vasculature leads to reduced blood flow, heterogeneous vessel wall and interstitial hypertension and results in inefficient delivery of cytotoxic drugs into tumors. The uptake of cytotoxic drugs [91] in tumors is primarily determined by the total number of functional blood vessels inside the tumor and the transport efficiency of each individual vessel. The treatment of anti-angiogenic agents dynamically affects the tumor vasculature and the consequent cytotoxic drugs transport into solid tumors. Besides, the optimal dosing and scheduling of angiogenesis inhibitors when combining with cytotoxic drugs are critical for the effect of angiogenesis inhibitors on cytotoxic drug penetration and efficacy, as extensively suppression of tumor vessels may ultimately reduce the cytotoxic drug penetration and efficacy. Indeed, improved clinical responses were observed when conventional chemotherapy was combined with low dose BEV as compared to high dose BEV [98]. Improved intratumoral cytotoxic drug concentration and penetration were also observed when given low dose of sunitinib as compared to high dose sunitinib [51,148]. In addition, chronic treatment with antiangiogenesis therapy eventually reduces tumor blood perfusion and increases tumor hypoxia in experimental animal studies [150, 151], indicating that uninterrupted treatment with the anti-angiogenic drug, although perhaps maximally effective as a monotherapy, may not be optimal for tumor vascular normalization-enhanced combination chemotherapy. Furthermore, as Jain [14] proposed, either less effect or over pruning of tumor vessels by angiogenesis inhibitors leads to no change on or reduced total functional blood vessels in tumors and resulting in no change on or decreased cytotoxic drugs transport. Even in the tumor vasculature normalization window, decreases in vessel permeability to cytotoxic drugs may overwhelm the favorable changes in vessel morphology for drug penetration and result in reduced drug levels in tumors as evidenced and discussed by Devineni [145]. Only the favorable effects on drug transport overweighting the unfavorable effects will result in an increase in drug transport capacity of the tumor vasculature and microenvironment. Increasing the total number of functional blood vessels, increasing the blood perfusion and decreasing the interstitial pressure are examples of the favorable effects, while reducing the blood vessel permeability and decreasing the total blood vessels are examples of the unfavorable effects. The observation of the decreased intratumoral TMZ concentration and penetration by Gallo et al. in rat glioma model is correlated to the decreased MVD in tumor. Thus the decreased intratumoral concentration of TMZ may due to the 
over-pruned microvessel [146, 147]. In contrast, the increased intratumoral TMZ concentration and penetration is significantly correlated to the VNI of the tumor [51, 148]. This increased intratumoral TMZ concentration is likely due to the proper tumor vasculature normalization. Thus defining the tumor vasculature normalization window and favorable effects of anti-angiogenic agents on the penetration of cytotoxic drugs into solid tumors is difficult, but crucial for the success of the combination of the anti-angiogenic agents and cytotoxic drugs regimen.

\subsubsection{The effects of BEV on cytotoxic drugs disposition in solid tumors}

No publications have estimated the effect of BEV on cytotoxic drug penetration in solid tumors for clinical patients, but several papers $[152,153]$ demonstrate that there is no pharmacokinetic interaction between BEV and cytotoxic drugs in the plasma of patients with solid tumors. Thus in this section, we will only discuss the effects of BEV on cytotoxic drugs disposition in solid tumors evaluated in preclinical studies.

In preclinical studies, Yanagisawa [49] demonstrated that the co-administration of BEV significantly increased the intratumoral paclitaxel concentration in both murine MX-1 human breast cancer and A547 human lung cancer xenograft models. Recently, We also evidenced that the intratumoral penetration of TPT was enhanced as much as $81 \%$ when given 1 to 3 days after BEV, compared with when both drugs were given concomitantly, or 7 days apart in neuroblastoma xenografts [154]. In consistent with this observation, tumor vasculature normalization was observed within 7 days after BEV administration and antitumor activity was also significantly enhanced when administering TPT 3 days after BEV compared to monotherapy or concomitant administration of the two drugs. The increased intratumoral penetration of TPT was closely associated with normalization of the tumor vasculature. Furthermore, Davidoff (St. Jude Children's Research Hospital, Memphis, TN) found that the tumor vasculature normalization window occurred between 2 to 5 days after BEV administration in the murine Rh30 rhabdomyosarcoma xenograft, and the treatment with ionizing radiation 2 or 5 days after $\mathrm{BEV}$ resulted in the greatest antitumor activity [155]. In order to further investigate whether there is a similar effect of BEV on TPT penetration in this Rh30 xenograft and whether an optional schedule of the BEV and TPT will lead to enhanced tumor inhibition, we proposed the current project to answer above questions and ultimately benefit children with rhabdomyosarcoma cancer by providing a better chemotherapy regimen. As discussed above, ARMS has a relatively low OS rate and is resistant to TPT treatment. If we can identify the optimal schedule of BEV and TPT, and enhance the intratumoral penetration and efficacy of TPT by using preclinical model, we may be able to design proper clinical trials to confirm the preclinical findings and improve the OS rate for children with ARMS. 


\subsection{Pharmacokinetic Models of TPT in Preclinical Studies}

Extensive preclinical pharmacokinetic studies for TPT have been published over the last 20 years [156-161]. Among these publications, numerous pharmacokinetic models either for in vitro intracellular TPT uptake and kinetics [156, 157] or for an in vivo pharmacokinetic model and effective schedule of TPT in human cancer xenografts [158-161] were proposed by several groups. Our discussion focuses mainly on TPT pharmacokinetic evaluation in preclinical animal models, including rodents and nonhuman primates.

In order to better define the pharmacokinetic behavior of TPT in both plasma and cerebrospinal fluid (CSF) and to measure TPT CSF penetration, Blaney et al. [159] performed a pharmacokinetic study of TPT in 3 adult male rhesus monkeys after an intravenous dose of $10 \mathrm{mg} / \mathrm{m}^{2}$ administered over 10 minutes and used a relative simplistic non-compartmental model to get the pharmacokinetic parameters. The CSF concentration peaked at 30 minutes after administration and the CSF penetration of TPT exceeded $30 \%$, which warranted the further study in patients with high risk or refractory central nervous system tumors. In a more pharmacokinetically elegant analysis, Balthasar's group from The State University of New York published two mathematical models of TPT in mice. The first one [158] described an integrated pharmacokinetic/toxicodynamic model to characterize the relationship between the TPT disposition and TPT induced toxicity. Body weight loss was used as the index of TPT-induced toxicity. The authors fitted four models composed of two disposition compartments and one peritoneal absorption compartment to the plasma concentration data, but with different kinetics of TPT absorption and elimination. A modified indirect response toxicity model was combined with the best fitting pharmacokinetic model selected from those four pharmacokinetic models. Four additional transit compartments were added to account for the delay of the time of maximum plasma TPT concentration and the time associated with the nadir body weight. The same group published a second paper regarding mathematical model of TPT in mice is a whole body physiologically based pharmacokinetic model to characterize and predict TPT concentrations in mouse plasma and tissues, such as lungs, heart, muscle, liver and brain [160].

Moreover, tremendous work on the pharmacokinetic study and mathematical model of TPT has been published from our laboratory [162-166]. The traditional pharmacokinetic model used in the lab to describe the plasma and target tumor drug concentration used a non-linear three compartmental model including central compartment, peripheral compartment and tumor compartment. In this model, plasma TPT concentration-time profile was fit to a two-compartment model using a maximum a posteriori (MAP) Bayesian algorithm as implemented in ADAPT 5[162, 167-169]. With the plasma pharmacokinetic parameters remaining fixed, a third compartment was added to represent the tumor disposition of TPT and the parameters describing the TPT tumor concentrations were estimated for each study by using the maximum likelihood approach. 


\subsection{Summary}

Although the overall survival rate for RMS is encouraging, the prognosis for patients with relapsed or metastatic RMS at diagnosis is very poor, especially for ARMS. Recently, antiangiogenesis agents have gained more attention due to their pivotal role in the modulation of tumor vasculature, especially RMS.

One of the biggest obstacles for chemotherapy is the insufficient delivery of cytotoxic drugs to their target tissue or tumor sites. The development of antiangiogenesis agents expands the treatment modality of chemotherapy. Tumor progression and metastases are highly dependent on angiogenesis to gain sufficient oxygen and nutrients. VEGF is a key regulator of tumor angiogenesis and anti-VEGF monoclonal antibody, $\mathrm{BEV}$, has shown enhanced anti-tumor activity in several tumor models when combined with conventional cytotoxic drugs. One of the mechanisms for the improved antitumor activity in combination therapy is BEV is able to normalize tumor vasculature by increasing the number of functional blood vessels in tumor, enhancing blood perfusion rate and decreasing interstitial pressure, and further increase the cytotoxic drug penetration into tumor. The schedule of combination therapy is crucial to catch the tumor vasculature normalization window and enhance the cytotoxic drug penetration. However, there is little known about the optimal schedule when BEV combining with conventional cytotoxic drugs.

TPT is one of the cytotoxic drugs used in patients with RMS. Like most other cytotoxic drugs, in order to exert its cytotoxic effect TPT has to reach tumor cells and further entering its targeting site-nucleus. The aberrant tumor vasculature, heterogeneous vessel walls and compacted extracellular space impedes TPT accessing tumor cells, resulting less drugs remaining to the targeting site. Our previous work showed that the intratumoral penetration of TPT was significantly enhanced after 3 day pre-treatment with BEV and the increased intratumoral penetration of TPT was closely associated with normalization of the tumor vasculature in neuroblastoma xenografts. In addition, BEV was shown to transiently normalize tumor vasculature in Rh30 RMS xenograft between 2 to 5 days. This work rationalizes the further evaluation of the time dependent effect of BEV on the penetration, pharmacokinetics and efficacy of TPT in Rh30 RMS xenograft.

In vivo microdialysis techniques are an invaluable tool for sampling unbound free drugs in tumor ECF and having the proper pharmacokinetic model is a pre-requisite to evaluate TPT penetration and pharmacokinetics in vivo. Combining microdialysis and pharmacokinetic modeling is an excellent way to assess the pharmacokinetics of drugs in target tissue. 


\subsection{Specific Aims}

Inhibition of VEGF can transiently normalize tumor vasculature and improve delivery of systemic chemotherapy. BEV, an anti-VEGF antibody, has been shown to transiently normalize tumor vasculature in an Rh30 orthotopic rhabdomyosarcoma xenograft model. However, the effects of BEV on the pharmacokinetics of TPT and the antitumor activity of TPT have not been evaluated in this tumor model.

Specific aim 1: determine whether TPT pharmacokinetics was dependent upon the schedule of BEV and TPT.

Specific aim 2: assess whether the combination and schedule of TPT and BEV contributed to differential antitumor activity in the Rh30 mouse model. 


\section{CHAPTER 2. THE EFFECT OF BEVACIZUMAB ON THE PHARMACOKINETICS OF TOPOTECAN IN A RH30 RHABDOMYOSARCOMA XENOGRAFT}

\subsection{Introduction}

In order to gain sufficient nutrients and oxygen for growth, tumor cells secret and recruit pro-angiogenic factors for the creation and maintenance of the vascular network in solid tumors [14]. As a result, the tumor vasculature is chaotic, the blood vessel walls are heterogeneous and the microenvironment is compact and has an interstitial hypertension and aberrant metabolic environment-all of which can be restored by anti-angiogenic treatments $[6,31,53,170]$. VEGF is a key regulator of tumor angiogenesis $[65,66,69]$. It helps recruit of bone-marrow-derived progenitor cells to the primary and metastatic sites to form a new vascular network to stimulate tumor growth. In addition, VEGF not only promotes proliferation, migration, and invasion of endothelial cells to maintain tumor vessel growth, but also inhibits endothelial cell apoptosis to facilitate tumor vessel survival. Furthermore, the overexpression of VEGF is frequently observed in human solid tumors, which is found to correlate with the extent of tumor angiogenesis, progression and survival in patients $[67,68]$. Thus, inhibiting VEGF represents a rational strategy in treating various malignant tumors.

Rhabdomyosarcoma is the most common soft tissue sarcoma among children 0-14 years, representing nearly 50\% of soft tissue sarcomas [171]. The two most common pathologic types of RMS are embryonal RMS (ERMS) and alveolar RMS (ARMS) with differential incidence, age pattern and body sites of occurrence [102]. ARMS grow more aggressively than ERMS and require more intensive treatment. Therefore they have a higher relapse incidence and worse prognostic outcome [109]. The 5 year failure-free survival rate for ARMS (65\%) is much lower than ERMS (82\%) [172]. In addition, 30\% of young patients experience recurrence and $50 \%$ to $95 \%$ of these patients die of progressive disease, even with intensified treatment [112]. So developing new agents or new strategies to increase current agent efficacy is urgent for the treatment of RMS, especially ARMS.

Bevacizumab (BEV) is a humanized monoclonal antibody that inhibits VEGF and currently used to treat various cancers, including colorectal, lung, breast, kidney cancers, and glioblastoma [173]. It is also in clinical trials for the treatment of RMS in combination with traditional cytotoxic chemotherapy $[174,175]$. However, the effect of dosing schedule of BEV in combination with cytotoxic drugs is not well understood. The Rh30 cell line retains the histologic appearance of ARMS [176]. VEGF is over-expressed in the Rh30 cell line and anti-VEGF treatment has been shown to inhibit the growth of Rh30 RMS xenograft [115]. The Rh30 cell line is more sensitive to Topotecan (TPT) than RD cell line, characterized as ERMS cell line [116]. TPT has been actively investigated in clinical trials and had an encouraging response rate in ARMS patients [177]. However, preclinical and clinical studies showed that RMS is resistant to TPT as 
most cytotoxic drugs $[118,119]$. One approach to overcome the drug resistant and increase its efficacy is to increase its intratumoral concentration of TPT.

Our previous work [154] has shown that BEV enhanced the penetration of TPT in orthotopic neuroblastoma xenografts possibly due to the transiently normalized tumor vasculature. In addition, BEV [155] has been shown to transiently normalize tumor vasculature in murine Rh30 rhabdomyosarcoma xenograft. Therefore, we hypothesized that the normalized tumor vasculature by BEV would facilitate the penetration of TPT in Rh30 RMS xenograft. The specific aim 1 of this project was to determine whether TPT pharmacokinetics was dependent upon the schedule of BEV and TPT. The specific aim 2 was to assess whether the combination and schedule of TPT and BEV contributed to differential antitumor activity in the Rh30 mouse model.

\subsection{Materials and Methods}

\subsubsection{Materials and chemicals}

TPT (Hycamtin, GlaxoSmithKline, Philadelphia, PA) was prepared in sterile saline for injection at a concentration of $0.4 \mathrm{mg} / \mathrm{ml}$. BEV (Avastin, Genentech, South San Francisco, CA) was diluted in sterile saline right before injection from stock concentration of $25 \mathrm{mg} / \mathrm{ml}$ to $1 \mathrm{mg} / \mathrm{ml}$. Isoflurane (Forane) was purchased from Baxter Pharmaceutical Products (New Providence, NJ). Acetonitrile and triethylamine were of high-performance liquid chromatography (HPLC) grade. All other chemicals and solvents used were of analytic grade or better.

\subsubsection{Cell culture}

The Rh30 pediatric RMS cell line was kindly provided by Dr. Davidoff (Department of Surgery, St. Jude Children's Research Hospital) and was grown as a monolayer in RPMI 1640 medium (Life Technologies Inc., Gaithersburg, MD) supplemented with $10 \%$ fetal bovine serum. When cells reached $80-90 \%$ confluency, they were collected, counted, and resuspended in phosphate-buffered saline (PBS) solution to prepare a cell suspension of $1 \times 10^{4}$ cells $/ \mu \mathrm{L}$ for tumor inoculation.

\subsubsection{Animals}

4 to 6-week-old female CB-17 SCID mice were purchased from Charles River Laboratories (Wilmington, MA). The mice were maintained on a 12 hour light/dark cycle with access to food and water ad libitum. All procedures were performed in accordance with a protocol approved by the Institutional Animal Care and Use Committee at St Jude Children's Research Hospital. 


\subsubsection{Tumor model and treatment}

Orthotopic ARMS xenograft was established as previously described by Dr. Davidoff [155]. Briefly, $2 \times 10^{6}$ Rh30 tumor cells suspended in $200 \mu \mathrm{L}$ PBS were injected into the right calf muscle of female CB-17 SCID mice. Intramuscular tumor size was estimated by measuring the size of the normal left calf and subtracting that volume $\left(\right.$ width $^{2} \times$ length $\times 0.5$ ) from the volume of the tumor-injected right calf.

Microdialysis studies were performed to address the specific aim 1. After approximately 4 weeks of tumor growth, mice were divided into 4 groups of equivalent tumor burden $\left(\sim 700 \mathrm{~mm}^{3}\right)$ and treatment was initiated. Six to eight mice per group were pretreated with either vehicle control or a single dose of $5.0 \mathrm{mg} / \mathrm{kg}$ BEV intravenously at 1 hour (vehicle control), 1 day, 3 days or 7 days before the tail vein injection of TPT (2.0 $\mathrm{mg} / \mathrm{kg}$ ). Using a previously described limited sampling strategy [178], we bled each mouse from the retro-orbital plexus 15, 60, and $180 \mathrm{~min}$ after the IV injection of TPT to assay total TPT in plasma. Tumor extracellular fluids were collected up to 6 hours by microdialysis. For TPT the pharmacokinetics studies after multiple doses of BEV administration, treatments were initiated when the tumor burden approached approximately $200 \mathrm{~mm}^{3}$. Six to eight mice per group were treated with BEV or vehicle twice weekly for total 4 doses before TPT administration. TPT was injected 1 hour (vehicle control), 1 day, 3 days or 7 days after the last dose of vehicle or BEV administration. Plasma was collected at 15,60 and 180 min after TPT IV injection and tumor extracellular fluids were collected up to 6 hours by microdialysis.

The antitumor activity after SDBT and MDBT were evaluated to address the specific aim 2. In the SDBT groups, a cohort of tumor-bearing mice was divided into 4 groups when the tumor burden was approximately $700 \mathrm{~mm}^{3}$. Five to six mice were treated with BEV only, TPT only, BEV given 1 day before TPT and BEV given 7 days before TPT. The final tumor volume in mice treated with BEV only and received TPT 1 day or 7 days after BEV were evaluated to assess whether the addition of TPT had effect on antitumor activity of BEV. Similarly, the final tumor volume in mice treated with TPT only and received TPT 1 day or 7 days after BEV were evaluated to assess whether the pre-treatment of BEV has effect on antitumor activity of TPT. Furthermore, the final tumor volume in mice treated with TPT 1 day and 7 days after BEV administration were evaluated to assess whether the antitumor activity was dependent upon the schedule of BEV and TPT. Nonparametric student's t-test was used for two group comparisons. Comparison of more than two groups was done by one way ANOVA and followed by Newman Keuls multiple comparison post hoc procedure if there was significant difference between groups. In the MDBT groups, the same cohorts of mice were used for the pharmacokinetics studies and the efficacy studies. An additional BEV only group was added for the efficacy study as monotherapy control. As the tumor burden was approximately $200 \mathrm{~mm}^{3}$, six to eight mice treated with BEV only, TPT only, TPT given 1 day, 3 days or 7 days after the last dose of BEV were evaluated for the efficacy studies. The final tumor volume in mice treated with BEV only and received TPT 1 day, 3 days or 7 days after the last dose BEV were evaluated to assess whether the addition of TPT has effect on antitumor activity of BEV. Similarly, the final tumor volume in mice treated 
with TPT only and received TPT 1 day, 3 days or 7 days after the last dose of BEV were evaluated to assess whether the pre-treatment of BEV has effect on antitumor activity of TPT. In addition, the final tumor volume in mice treated with TPT 1 day, 3 days or 7 days after the last dose of BEV administration were evaluated to assess whether the antitumor activity was dependent upon the schedule of BEV and TPT.

Collectively, the TPT penetration and efficacy study designs were shown in Tables 1-2 and Figures 1-2.

\subsubsection{In vivo tumor microdialysis}

The principle and methodology of microdialysis sampling have been reviewed thoroughly in volume 45, issue 2-3 of Advanced Drug Delivery Reviews. In brief, a short length of semi-permeable microdialysis probe is implanted into tissue and continuously perfused with a physiologic solution at a low flow rate $(0.5-10 \mu 1 / \mathrm{min})$. The analyte of interest in the tissue ECF diffuses into the microdialysis probe, resulting from the concentration gradient across the probe membrane, and is carried via microtubing into the collection vials.

In the present study, a CMA/20 microdialysis probe (CMA Microdialysis, Chelmsford, MA) was used. The probe was perfused with Ringer's solution (USP) at 0.5 $\mu \mathrm{L} / \mathrm{min}$ flow rate by a CMA 102 pump (CMA Microdialysis, Chelmsford, MA). The probe was equilibrated for one hour prior to the microdialysis sample collection. The dialysate samples were collected up to 6 hours every 30 minutes for the off line system and every 18 minutes for the online system [179] after TPT injection. The off line system was composed of two steps-collecting samples manually and analyzing samples on a separate HPLC system, while the samples were directly injected into a connected HPLC system and analyzed at real-time on the on line system [179]. The samples were stored in the $-80^{\circ} \mathrm{C}$ freezer before analysis for the off line system.

For each microdialysis experiment, retrodialysis calibration was performed as previously described [178] after the sample collection. Briefly, a TPT solution (50 $\mathrm{ng} / \mathrm{mL}$ ) was prepared in Ringer's solution and perfused through the microdialysis probe at two different flow rates 4.0 and $0.5 \mu \mathrm{L} / \mathrm{min}$. The system was allowed to equilibrate for 30 minutes between the flow rate change. The total TPT concentration in the solution exiting the probe at $4.0 \mu \mathrm{L} / \mathrm{min}(C$ in) and at $0.5 \mu \mathrm{L} / \mathrm{min}$ (Cout) was determined by HPLC. The recovery was estimated as shown in Equation 1.

$$
\text { Recovery } \%_{\mathrm{TPT}}=\frac{\mathrm{C}_{\text {in }}-\mathrm{C}_{\text {out }}}{\mathrm{C}_{\mathrm{in}}} \times 100
$$

At the end of the retrodialysis study the mice were euthanized by cervical dislocation under anesthesia. 
Table 1. TPT penetration study design after a single dose of BEV treatment (SDBT)

\begin{tabular}{ccccc}
\hline Groups & T & 1D BT & 3D BT & 7D BT \\
\hline BEV & Vehicle IV & Single IV & Single IV & Single IV \\
$(5 \mathrm{mg} / \mathrm{kg})$ & Day 0 & Day 0 & Day 0 & Day 0 \\
$\mathrm{TPT}$ & Single IV & Single IV & Single IV & Single IV \\
$(2 \mathrm{mg} / \mathrm{kg})$ & Day 0 & Day 1 & Day 3 & Day 7 \\
\hline
\end{tabular}

Treatment was initiated when tumor burden was approaching approximately $700 \mathrm{~mm}^{3}$ and four groups were evaluated: group $\mathrm{T}$ received a single dose of vehicle 1 hour before TPT; group 1D BT received a single dose of BEV 1 day before TPT; group 3D BT received a single dose of BEV 3 days before TPT; group 7D BT received a single dose of BEV 7 days before TPT.

Table 2. TPT penetration study design after multiple doses of BEV treatment (MDBT)

\begin{tabular}{ccccc}
\hline Groups & mT & 1D mBT & 3D mBT & 7D mBT \\
\hline & Vehicle IV & Multiple IV & Multiple IV & Multiple IV \\
BEV & Day -10, -7, & Day -10, -7, & Day -10, -7, & Day -10, -7, \\
$(5 \mathrm{mg} / \mathrm{kg})$ & $-3,0$ & $-3,0$ & $-3,0$ & $-3,0$ \\
$\mathrm{TPT}$ & Single IV & Single IV & Single IV & Single IV \\
$(2 \mathrm{mg} / \mathrm{kg})$ & Day 0 & Day 1 & Day 3 & Day 7 \\
\hline
\end{tabular}

Treatment was initiated when tumor burden was approaching approximately $200 \mathrm{~mm}^{3}$ and four groups were evaluated: group $\mathrm{mT}$ received four doses of vehicle and TPT was given 1 hour after the last dose of vehicle; group 1D mBT received four doses of BEV and TPT was given 1 day after the last dose of BEV; group 3D $\mathrm{mBT}$ received four doses of BEV and TPT was given 3 days after the last dose of BEV; group 7D mBT received four doses of BEV and TPT was given 7 days after the last dose of BEV. 


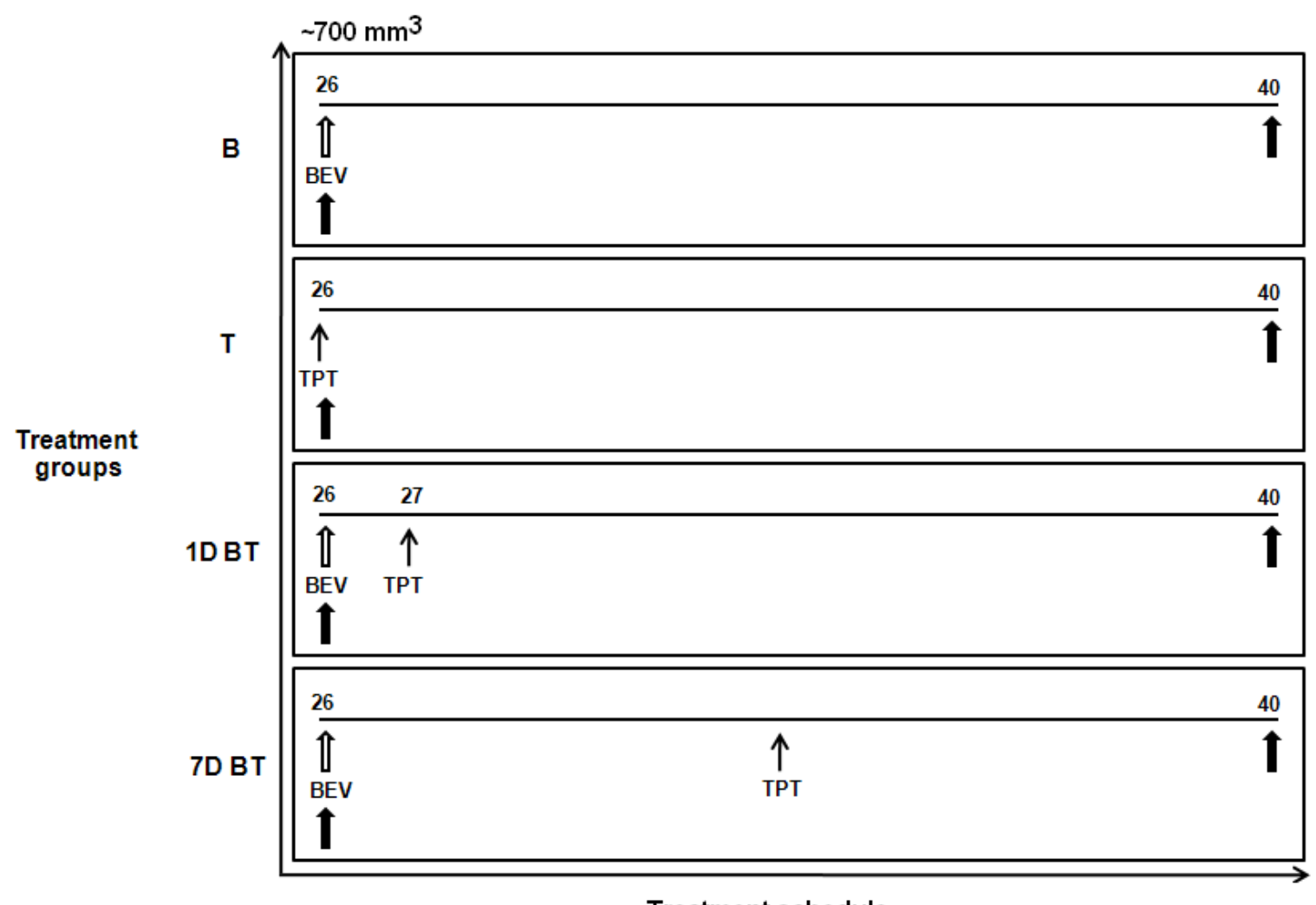

Treatment schedule

Figure 1. TPT efficacy study design after SDBT

Treatment was initiated when tumor burden was approaching approximately $700 \mathrm{~mm}^{3}$. Group B received a single dose of BEV and groups T, 1D BT and 7D BT received some therapeutic regimen as Table 1. Tumor volume was measured at the beginning of the

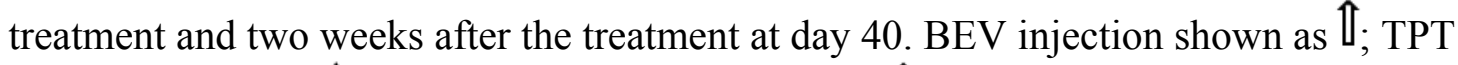
injection shown as $\uparrow$; tumor measurements shown as $\mathbf{I}$. 


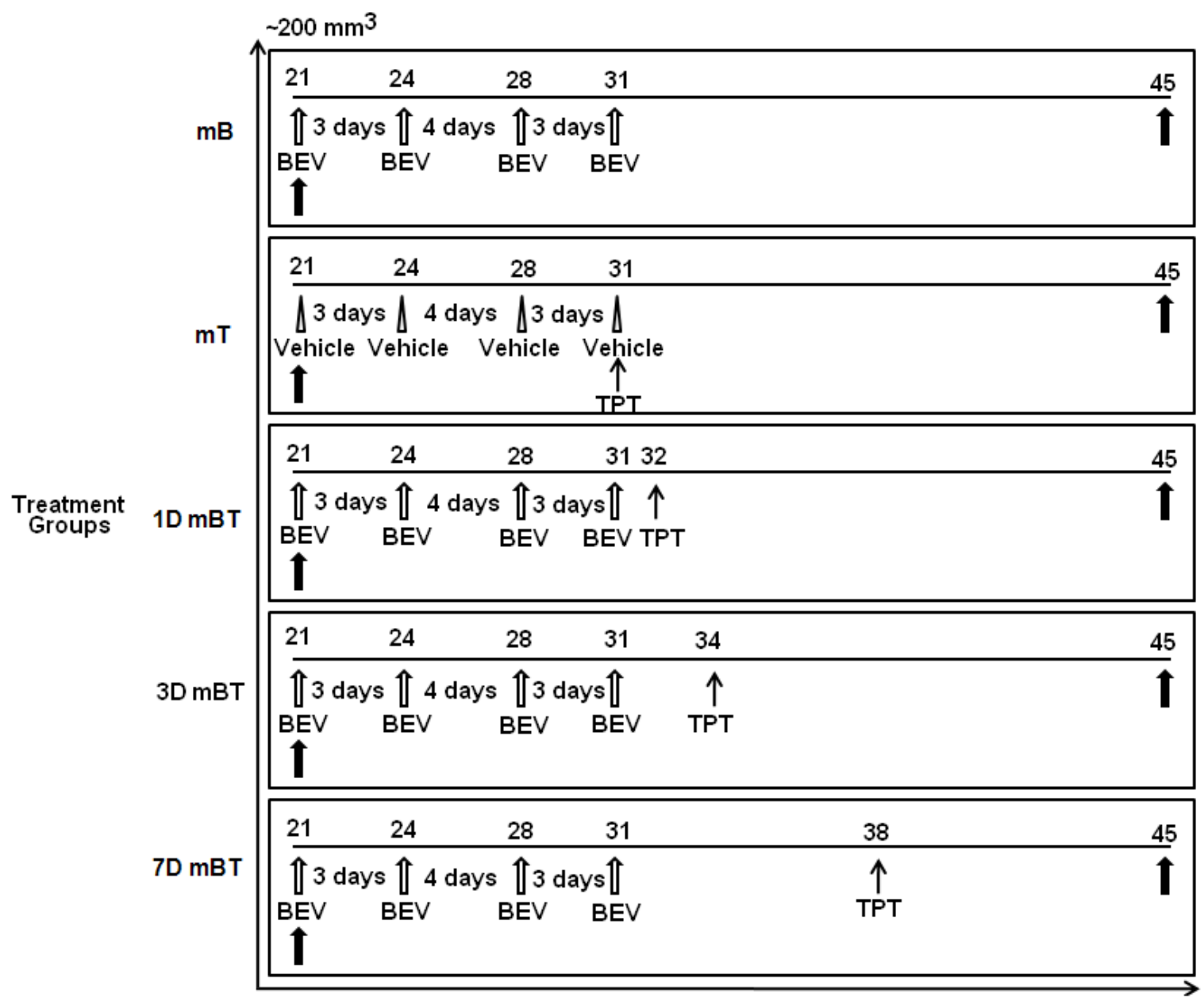

Treatment schedule

Figure 2. TPT penetration plus efficacy study design after MDBT

Treatment was initiated when tumor burden was approaching approximately $200 \mathrm{~mm}^{3}$. Group $\mathrm{mB}$ received four doses of BEV only and all other groups are the same as in Table 2. Microdialysis study was performed on the day of TPT administration and tumor volume was measured at the beginning of the treatment and 24 days after the treatment. BEV injection shown as $\Uparrow$; vehicle injection shown as $\triangle$; TPT injection shown as $\uparrow$; tumor measurements shown as $\mathbf{T}$. 


\subsubsection{High-performance liquid chromatography analysis for PK studies}

Total TPT in plasma and tumor ECF were measured as previously described [165, 180]. $20 \mu \mathrm{L}$ plasma aliquots were added to $80 \mu \mathrm{L}$ cold methanol $\left(-30^{\circ} \mathrm{C}\right)$. Samples were vortex mixed vigorously and centrifuged at $10,000 \mathrm{rpm}$ for 2 minutes. TPT in the plasma methanolic supernatants and tumor ECF were converted to total TPT by adding five parts methanolic supernatant or tumor ECF to one part $20 \%$ phosphoric acid and injected into HPLC with fluorescence detection. The unbound plasma TPT concentration was calculated on the basis of a previous study [178], showing a $30.1 \%$ unbound fraction in CB-17 SCID mice. All TPT concentrations reported in this study were the total unbound TPT concentration.

\subsubsection{Pharmacokinetic model evaluation}

Different PK models were considered to describe the TPT concentrations in plasma and tumor ECF: (1) a three compartmental PK model previously used in our lab (Figure 3) [162, 165, 169]; (2) we also evaluated other multi-compartmental models during the data analysis. The final modified multi-compartmental PK model is shown in Figure 4.

The modified multi-compartmental PK model significantly improved the estimation of PK parameters describing TPT in tumor compartment compared to the conventional model. This general model can be applied for drug administration via orally, IP or IV bolus. The model has an absorption compartment, a central and a peripheral compartment, and a tumor compartment. Additionally, two transient compartments between the plasma and tumor compartments were incorporated to account for the delay of drug transport from plasma to tumor compartment. The differential equations are shown in Equations 2-7. Model parameters estimated for intravenous dosing included elimination rate constants (Ke is systemic elimination rate constant; Kcp, Kpc and Kct are intercompartment rate constants; Kte is elimination rate constant for TPT leaving tumor compartment) and the volume of distribution in central compartment ( $\mathrm{Vc}$ ) and tumor compartment $(\mathrm{Vt})$. The secondary parameters were determined: $\mathrm{CLin}=\mathrm{Kct} \times \mathrm{Vc}$ and CLout $=$ Kte $\times$ Vt. The independent rate constants between the transient compartments were not identifiable and were set to the elimination rate constant Kct. All volume units are described in liters $/ \mathrm{m}^{2}$ and all elimination rates are described in hour ${ }^{-1}$.

$$
\begin{array}{cc}
\frac{d X_{1}}{d t}=-\left(K_{e}+K_{c p}+K_{c t}\right) \times X_{1}+K_{a} \times X_{2}+K_{p c} \times X_{3}+R_{(1)} & \text { Eq. } 2 \\
\frac{d X_{2}}{d t}=-K_{a} \times X_{2} & \text { Eq. } 3 \\
\frac{d X_{3}}{d t}=-K_{p c} \times X_{3}+K_{c p} \times X_{1} & \text { Eq. } 4 \\
\frac{d X_{4}}{d t}=-K_{c t} \times X_{4}+K_{c t} \times X_{1} & \text { Eq. } \mathbf{5}
\end{array}
$$




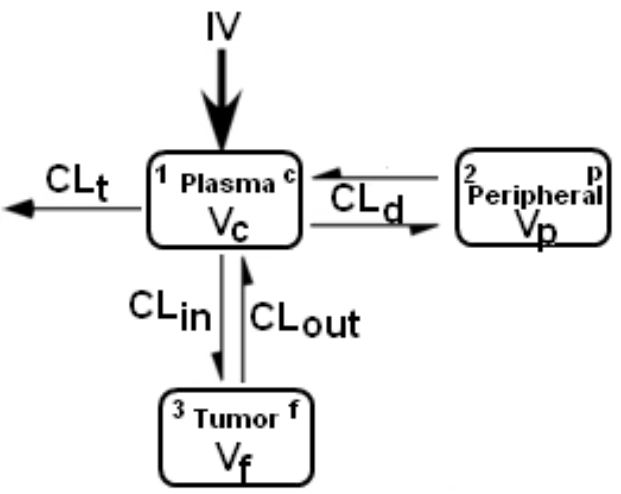

Figure 3. A pharmacokinetic model for TPT

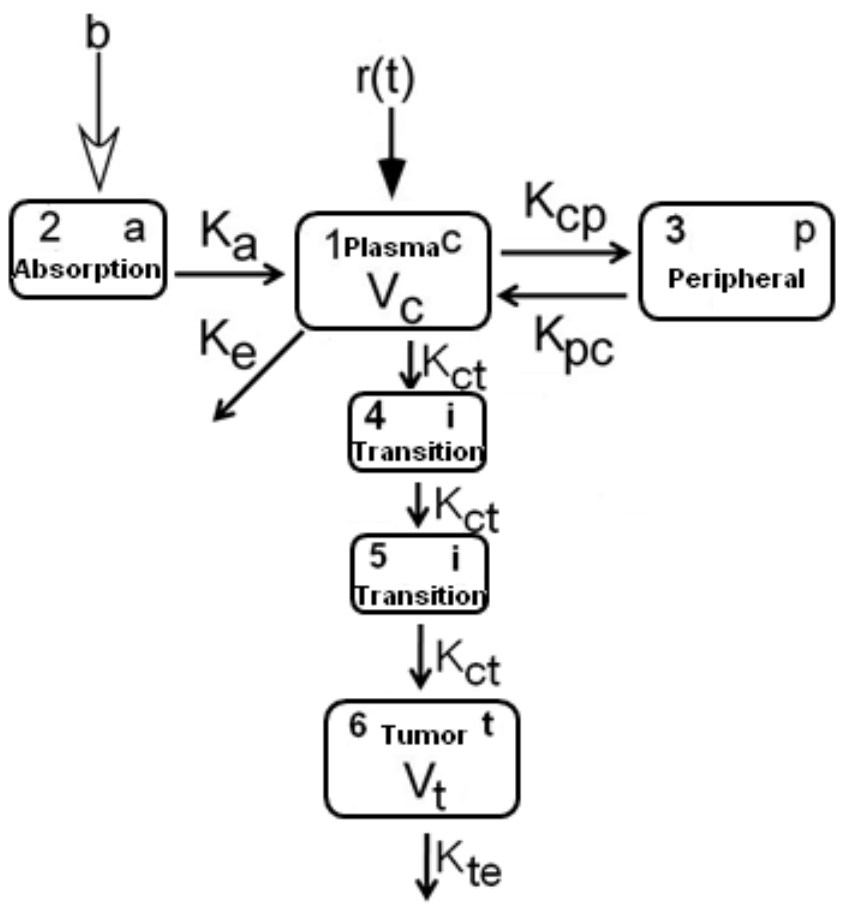

Figure 4. The modified multi-compartmental PK model for TPT 


$$
\begin{array}{lr}
\frac{d X_{5}}{d t}=-K_{c t} \times X_{5}+K_{c t} \times X_{4} & \text { Eq. } 6 \\
\frac{d X_{6}}{d t}=-K_{c t} \times X_{5}+K_{t e} \times X_{6} & \text { Eq. } 7
\end{array}
$$

The variables $\mathrm{X}_{1}, \mathrm{X}_{2}, \mathrm{X}_{3}, \mathrm{X} 4, \mathrm{X} 5$ and $\mathrm{X} 6$ are to the drug amounts in the central, absorption, peripheral, two transient compartments and tumor compartments; Ke is systemic elimination rate constant; Kcp and Kpc are intercompartment rate constants between peripheral and central compartments; Kct is elimination rate constant from central compartment to tumor compartment; Kte is elimination rate constant leaving from tumor compartment; $\mathrm{Vc}$ is volume of distribution in central compartment and $\mathrm{Vt}$ is volume of distribution in tumor compartment.

\subsubsection{Population pharmacokinetic analysis}

Non linear mixed effects modeling using the MLEM algorithm implemented in ADAPT 5 [181] was used to evaluate the TPT plasma and tumor ECF concentrations. In the MLEM algorithm, ML is combined with an EM algorithm. The EM algorithm consists of two steps. In the first step, each individual's parameters are estimated using the latest predicted parameter values and the observed data. In the second step, parameter values are updated to maximize the log-likelihood function in the first step. These two steps are then iterated until convergence. The initial values for population means and population covariance matrix (inter-individual variability) were estimated by naïve pooled analysis. Both population and individual estimates ware determined. The model-fitted curve for each mouse was used to estimate the area under the concentration-time curve from time zero to $6 \mathrm{hr}$ in plasma (AUCp) and tumor ECF (AUCt). The measures of penetration were expressed as AUC tumor-to-plasma ratio (AUCt/AUCp).

\subsubsection{Covariate analysis}

A diagnostic screening was done to identify covariates such as the presence of $\mathrm{BEV}$ in the treatment and the different schedules of the combination therapy that potentially affected TPT PK parameters, including systemic elimination rate Ke, elimination rate from tumor compartment Kte and volume of distribution in tumor compartment Vt. These potential covariates were then included in the non-linear mixed-effects population model to investigate their ability to significantly improve the model fit (by a reduction of at least $3.84[p<0.05]$ in the $-2 \log$-likelihood, on the basis of the $\chi^{2}$-test if the models differed in degree of freedom by 1 ). 


\subsubsection{Statistical analyses}

For each mouse, a TPT tumor-to-plasma AUC ratio was calculated and summary statistics (mean, standard deviation and the variation coefficient) were used to describe the TPT penetration results for each treatment group. Nonparametric student's t-test was used for two group comparisons. Comparison of more than two groups was done by one way ANOVA and followed by Newman Keuls multiple comparison post hoc procedure if there was significant difference between groups. $\mathrm{P}$ values less than 0.05 indicated statistical significance.

\subsection{Results}

\subsubsection{The effect of BEV on the pharmacokinetics of TPT}

We studied the intratumoral penetration of TPT after combining either single dose of BEV or multiple doses of BEV with different schedule. The TPT concentration-time profiles in plasma and tumor from representative mice is depicted in Figure 5 (SDBT) and Figure 6 (MDBT). The TPT AUCt, AUCp and AUCt/AUCp for each treatment group are shown in Table 3 and Figure 7 (SDBT) and Table 4 and Figure 8 (MDBT). One way ANOVA multiple group comparisons indicated that there was no significant difference in the plasma exposure between groups after SDBT $(p=0.5388)$ and in the intratumoral exposure, plasma exposure and intratumoral penetration of TPT between groups after MDBT ( $p=0.297, p=0.932$ and $p=0.307$, respectively). However, pre-treatment of single dose BEV showed a trend toward higher intratumoral exposure $(p=0.0610)$ and penetration $(p=0.0662)$ of TPT given 1 day after BEV administration compared to either TPT given alone or TPT given 3 days and 7 days after BEV. However, the power for distinguishing the intratumoral exposure and penetration of TPT between group T and group 1D BT at significance level 0.05 is as low as $6.97 \%$ and $31.6 \%$, while the power for distinguishing the intratumoral exposure and penetration of TPT between group T and group 7D BT at significance level 0.05 are $53.9 \%$ and $82.8 \%$.

The population PK parameters obtained for each treatment group are listed in Table 5 (SDBT) and Table 6 (MDBT). Based on visual inspection of the parameters, there is substantial variability in TPT pharmacokinetics between SDBT groups. As depicted in Figure 9, covariate analysis indicated that SDBT correlated with higher systemic elimination $(\mathrm{Ke})$ of topotecan and the elimination rate from tumor compartment (Kte) also associated to the different treatment schedule between TPT and BEV. One day SDBT significantly increased TPT elimination rate from tumor compartment and after 3 or 7 days' SDBT, TPT elimination rate from tumor compartment decreased to the level without SDBT. Specifically, when BEV was incorporated in the model for Ke, a significant reduction in negative log-likelihood $(-109)$ was observed $(p<0.0001)$ and the inter-individual variability for Ke decreased from $234 \%$ to $112 \%$. Similarly, when treatment regimen (T, 1D BT, 3D BT, and 7D BT) was considered as a covariate for Kte, negative log-likelihood reduced as much as $200(p<0.0001)$ and inter-individual 

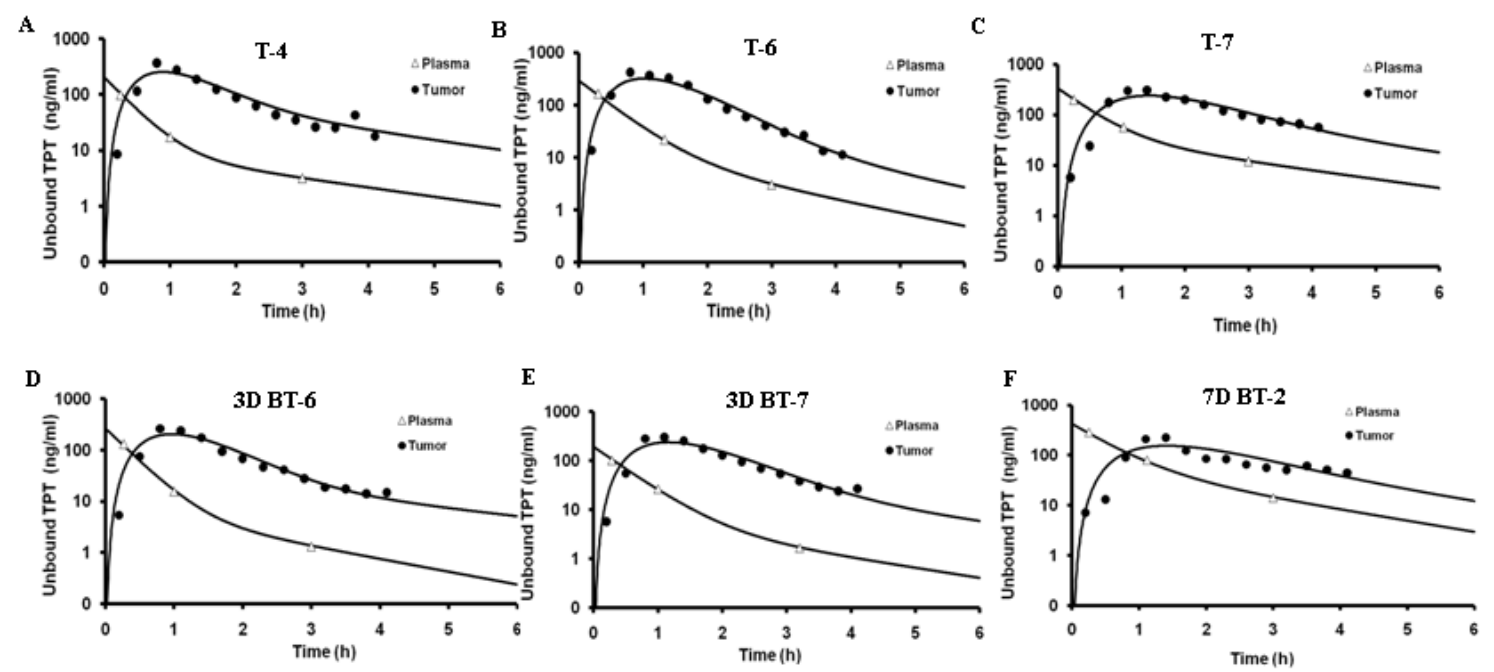

Figure 5. Representative plasma and tumor disposition of TPT with/without SDBT in mice bearing Rh30 RMS xenograft

Representative unbound TPT concentration-time plots in tumor ECF $(\bullet)$ and plasma $(\Delta)$ in Rh30 rhabdomyosarcoma xenograft mice were shown above. Compared to the three compartmental PK model, the modified multi-compartmental PK model significantly $(p<0.001)$ improved the model fit (Reduction in the -log-likelihood are 93.1, 57.2, 82.3, $114,35.9$ and 60.5 for T-4, T-6, T-7, 3D BT-6, 3D BT-7 and 7D BT-2, respectively). T-4, T-6 and T-7 are mice \#4, \#6 and \#7 in group T; 3D BT-6 and 3D BT-7 are mice \#6 and $\# 7$ in group 3D BT; 7D BT-2 is mouse \#2 in group 7D BT. 

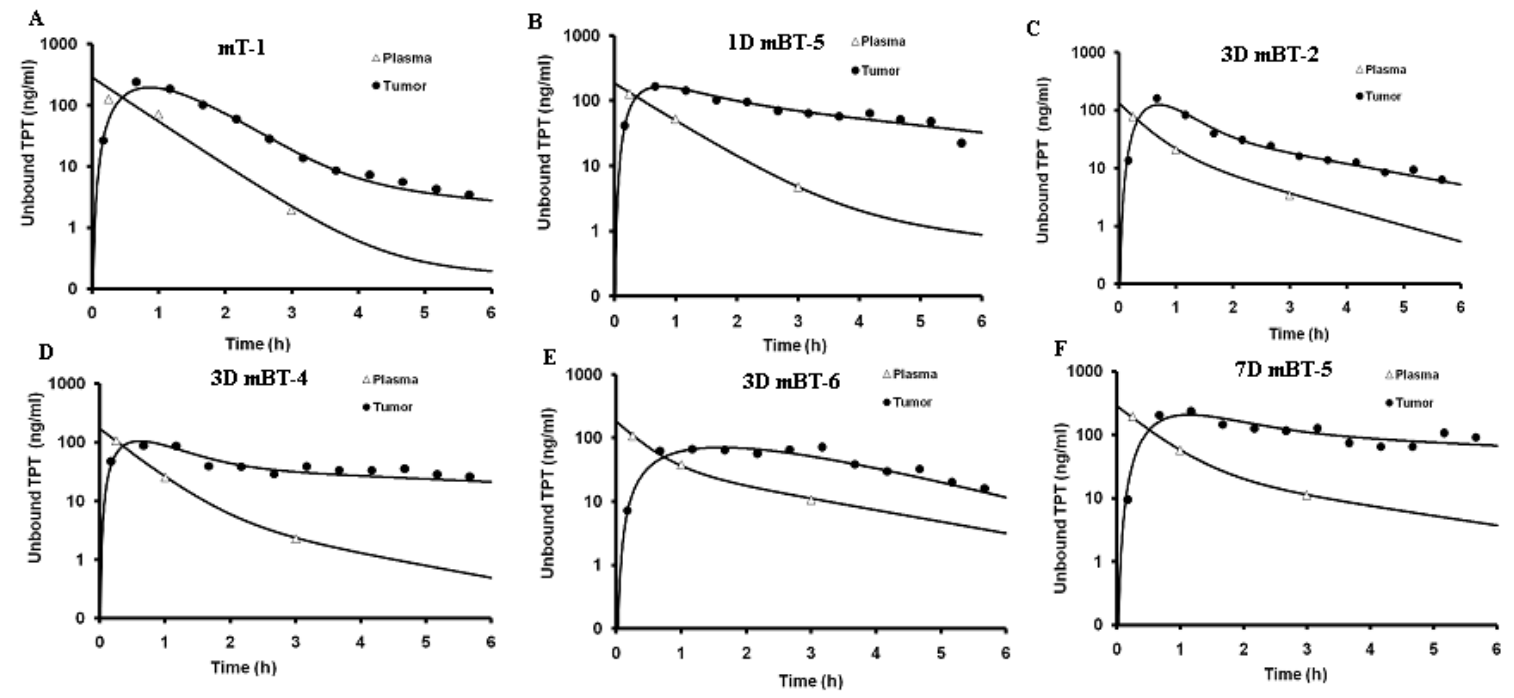

Figure 6. Representative plasma and tumor disposition of TPT with/without MDBT in mice bearing Rh30 RMS xenograft

Representative unbound TPT concentration-time plots in tumor ECF $(\mathbf{O})$ and plasma $(\triangle)$ in Rh30 rhabdomyosarcoma xenograft mice were shown above. Compared to the three compartmental PK model, the modified multi-compartmental PK model significantly $(p<0.05)$ improved the model fit (Reduction in the -log-likelihood are 42.5, 5.67, 42.5, 29.3, 9.37 and 16.8 for mT-1, 1D mBT-5, 3D mBT-2, 3D mBT-4, 3D mBT-6 and 7D $\mathrm{mBT}-5$, respectively). $\mathrm{mT}-1$ is mouse \#1 in group $\mathrm{mT}$; $1 \mathrm{D} \mathrm{mBT}-5$ is mouse \#5 in group 1D mBT; 3D mBT-2, 3D mBT-4 and 3D mBT-6 are mice \#2, \#4 and \#6 in group 3D $\mathrm{mBT}$; 7D mBT-5 is mouse \#5 in group 7D mBT. 
Table 3. TPT penetration after SDBT in mice bearing Rh30 RMS xenograft

\begin{tabular}{|c|c|c|c|c|c|c|c|c|c|}
\hline \multirow{2}{*}{$\begin{array}{c}\text { Parameters } \\
\text { Groups }\end{array}$} & \multicolumn{3}{|c|}{ AUCt (ng•hr/L) } & \multicolumn{3}{|c|}{ AUCp (ng•hr/L) } & \multicolumn{3}{|c|}{ AUCt/AUCp } \\
\hline & Mean & SD & CV\% & Mean & SD & CV\% & Mean & SD & CV\% \\
\hline $\mathbf{T}$ & 534 & 76.2 & 14.3 & 153 & 64.1 & 41.8 & 3.79 & 1.13 & 29.7 \\
\hline 1D BT & 553 & 169 & 30.6 & 110 & 16.8 & 15.2 & 4.93 & 1.09 & 22.0 \\
\hline 3D BT & 339 & 104 & 30.6 & 132 & 69.9 & 53.0 & 2.98 & 1.47 & 49.1 \\
\hline 7D BT & 353 & 62.6 & 17.7 & 184 & 101 & 54.7 & 2.17 & 0.700 & 32.4 \\
\hline
\end{tabular}

$\mathrm{SD}$ refers to standard deviation and $\mathrm{CV} \%$ refers to coefficient of variation $(\mathrm{CV} \%=\mathrm{SD} / \mathrm{Mean} \times \%)$. 


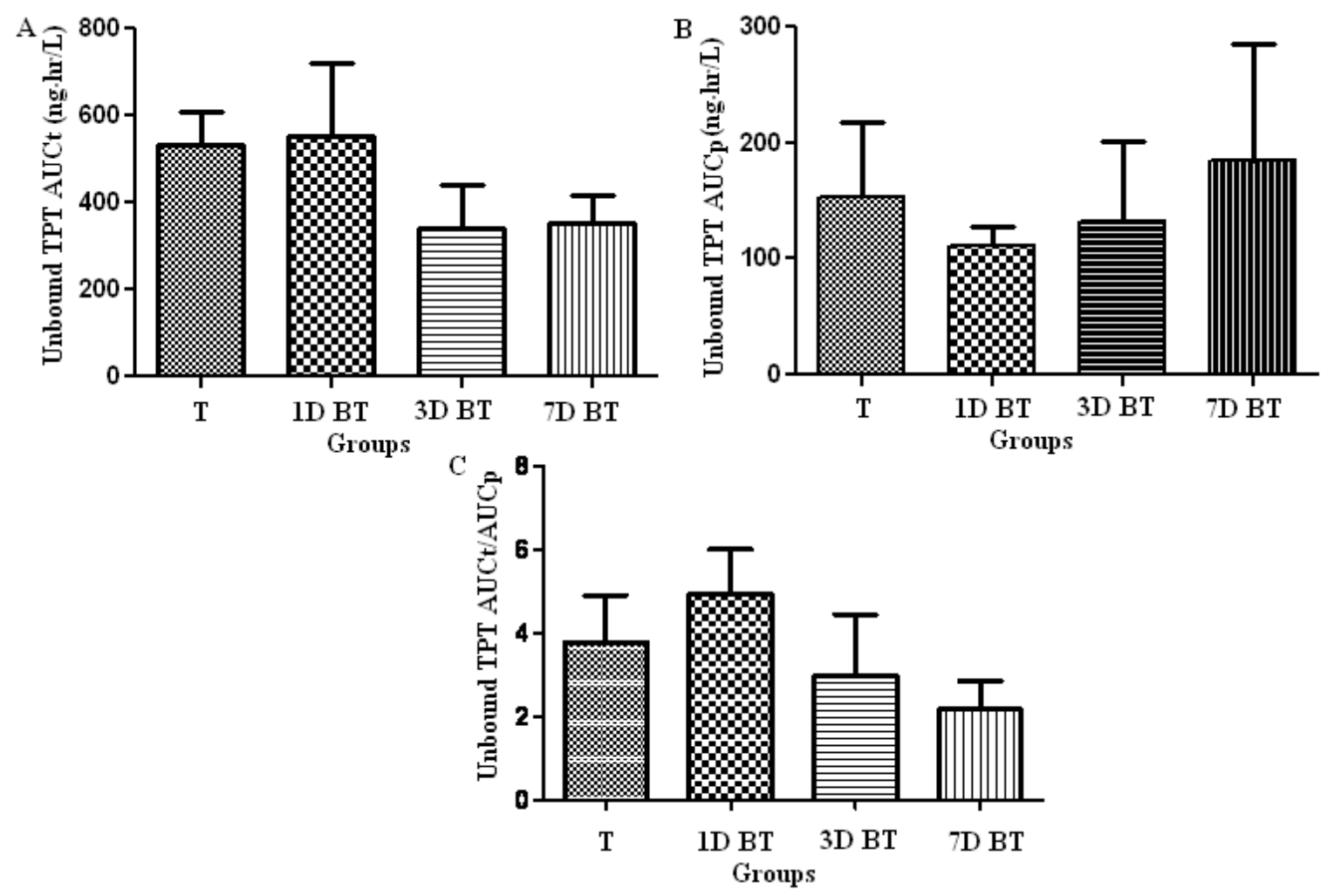

Figure 7. The effect of BEV on the intratumoral exposure, plasma exposure and intratumoral penetration of TPT after SDBT in mice bearing orthotopic Rh30 RMS xenograft

The intratumoral and plasma exposure of TPT is shown in Panel A and B. The penetration of TPT is shown in Panel C. One way ANOVA analysis: $p=0.0610$ for panel $\mathrm{A} ; p=0.539$ for panel $\mathrm{B}$ and $p=0.0662$ for panel $\mathrm{C}$ 
Table 4. TPT penetration after MDBT in mice bearing Rh30 RMS xenograft

\begin{tabular}{|c|c|c|c|c|c|c|c|c|c|}
\hline \multirow{2}{*}{$\begin{array}{c}\text { Parameters } \\
\text { Groups }\end{array}$} & \multicolumn{3}{|c|}{ AUCt (ng・hr/L) } & \multicolumn{3}{|c|}{ AUCp (ng•hr/L) } & \multicolumn{3}{|c|}{ AUCt/AUCp } \\
\hline & Mean & SD & $\mathrm{CV} \%$ & Mean & SD & $\mathrm{CV} \%$ & Mean & SD & CV\% \\
\hline $\mathbf{m T}$ & 428 & 77.1 & 18.0 & 165 & 28.4 & 17.2 & 2.68 & 0.859 & 32.0 \\
\hline $1 \mathrm{D} \mathrm{mBT}$ & 519 & 161 & 31.0 & 169 & 29.0 & 17.1 & 3.06 & 0.787 & 25.7 \\
\hline 3D mBT & 370 & 180 & 48.7 & 154 & 52.9 & 34.3 & 2.40 & 0.673 & 28.0 \\
\hline 7D mBT & 516 & 144 & 27.8 & 168 & 51.0 & 30.3 & 3.17 & 0.787 & 24.8 \\
\hline
\end{tabular}

$\mathrm{SD}$ refers to standard deviation and $\mathrm{CV} \%$ refers to coefficient of variation $(\mathrm{CV} \%=\mathrm{SD} / \mathrm{Mean} \times \%)$. 

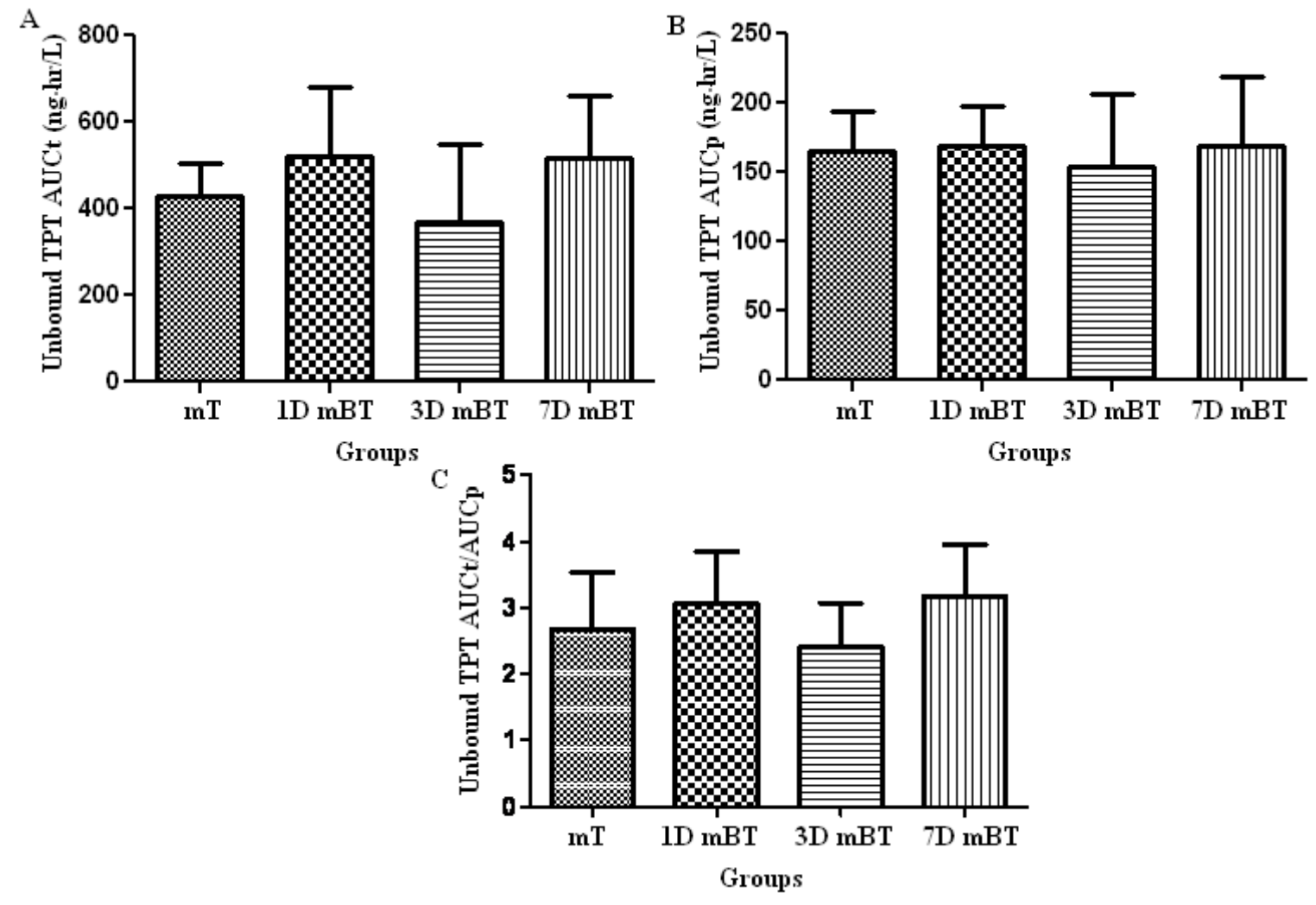

Figure 8. The effect of BEV on the intratumoral exposure, plasma exposure and intratumoral penetration of TPT after MDBT in mice bearing orthotopic Rh30 RMS xenograft

The intratumoral and plasma exposure of TPT is shown in Panel A and B. The penetration of TPT is shown in Panel C. One way ANOVA analysis: $p=0.297$ for panel $\mathrm{A} ; p=0.931$ for panel $\mathrm{B}$ and $p=0.307$ for panel $\mathrm{C}$ 


\section{Table 5. Population PK parameters of TPT estimated after SDBT}

\begin{tabular}{|c|c|c|c|c|c|c|c|c|c|c|c|c|}
\hline \multirow{2}{*}{$\begin{array}{c}\text { Groups \# } \\
\text { Parameters }\end{array}$} & \multicolumn{3}{|c|}{$\mathrm{T}$} & \multicolumn{3}{|c|}{ 1D BT } & \multicolumn{3}{|c|}{ 3D BT } & \multicolumn{3}{|c|}{$7 \mathrm{D} \mathrm{BT}$} \\
\hline & Mean & SD & IIV & Mean & SD & IIV & Mean & SD & IIV & Mean & SD & IIV \\
\hline $\operatorname{Ke}(1 / \mathrm{h})$ & 0.448 & 0.120 & 26.7 & 1.51 & 0.760 & 50.3 & 1.07 & 0.819 & 76.6 & 1.06 & 0.804 & 76.0 \\
\hline $\mathrm{Vc}\left(\mathrm{L} / \mathrm{m}^{2}\right)$ & 14.1 & 3.71 & 26.2 & 12.7 & 6.66 & 52.4 & 13.7 & 9.33 & 68.2 & 12.6 & 6.93 & 54.8 \\
\hline $\operatorname{Kcp}(1 / h)$ & 0.627 & 0.448 & 71.4 & 1.59 & 1.57 & 99.2 & 1.32 & 1.33 & 100 & 0.604 & 0.700 & 116 \\
\hline $\mathrm{Kpc}(1 / \mathrm{h})$ & 0.588 & 0.0600 & 10.2 & 0.344 & 0.0987 & 28.6 & 0.791 & 0.631 & 79.7 & 0.667 & 0.0600 & 9.00 \\
\hline $\operatorname{Kct}(1 / \mathrm{h})$ & 2.11 & 0.395 & 18.7 & 1.48 & 0.288 & 19.5 & 1.83 & 0.706 & 38.6 & 1.42 & 0.194 & 13.6 \\
\hline Kte $(1 / \mathrm{h})$ & 4.61 & 1.04 & 22.5 & 16.3 & 7.07 & 43.4 & 6.03 & 3.56 & 59.1 & 6.45 & 0.500 & 7.74 \\
\hline $\mathrm{Vt}\left(\mathrm{L} / \mathrm{m}^{2}\right)$ & 2.12 & 0.239 & 11.3 & 0.307 & 0.173 & 56.4 & 1.78 & 1.36 & 76.6 & 1.50 & 0.399 & 26.6 \\
\hline
\end{tabular}

MLEM program was used in this population modeling for each individual group. Mean and SD refer to population mean and population standard deviation; IIV refers to inter-individual variability as of $\% \mathrm{CV}$. 
Table 6. $\quad$ Population PK parameters of TPT estimated after MDBT

\begin{tabular}{|c|c|c|c|c|c|c|c|c|c|c|c|c|}
\hline \multirow{2}{*}{$\begin{array}{c}\text { Groups \# } \\
\text { Parameters }\end{array}$} & \multicolumn{3}{|c|}{$\mathrm{mT}$} & \multicolumn{3}{|c|}{$1 \mathrm{D} \mathrm{mBT}$} & \multicolumn{3}{|c|}{ 3D mBT } & \multicolumn{3}{|c|}{ 7D mBT } \\
\hline & Mean & $\mathrm{SD}$ & IIV & Mean & $\mathrm{SD}$ & IIV & Mean & SD & IIV & Mean & $\mathrm{SD}$ & IIV\% \\
\hline $\operatorname{Ke}(1 / \mathrm{h})$ & 0.825 & 0.505 & 61.2 & 0.371 & 0.323 & 87.0 & 0.284 & 0.211 & 74.4 & 0.343 & 0.392 & 114 \\
\hline $\mathrm{Vc}\left(\mathrm{L} / \mathrm{m}^{2}\right)$ & 9.61 & 2.22 & 23.1 & 8.81 & 2.24 & 25.5 & 11.2 & 5.01 & 44.6 & 10.8 & 7.97 & 73.7 \\
\hline $\operatorname{Kcp}(1 / h)$ & 1.35 & 1.61 & 119 & 4.93 & 1.30 & 26.3 & 4.06 & 1.43 & 35.3 & 1.88 & 2.82 & 150 \\
\hline Kpc $(1 / h)$ & 0.795 & 0.538 & 67.7 & 0.966 & 0.471 & 48.8 & 1.14 & 0.891 & 78.1 & 0.602 & 0.383 & 63.6 \\
\hline $\operatorname{Kct}(1 / \mathrm{h})$ & 1.93 & 0.532 & 27.6 & 1.68 & 0.630 & 37.4 & 2.10 & 0.842 & 40.2 & 1.66 & 0.995 & 60.0 \\
\hline Kte $(1 / \mathrm{h})$ & 15.8 & 10.1 & 63.9 & 11.3 & 8.54 & 75.4 & 15.3 & 18.9 & 124 & 14.0 & 10.7 & 76.2 \\
\hline $\mathrm{Vt}\left(\mathrm{L} / \mathrm{m}^{2}\right)$ & 0.580 & 0.467 & 80.5 & 0.611 & 0.549 & 89.8 & 0.791 & 1.13 & 143 & 0.501 & 0.424 & 84.6 \\
\hline
\end{tabular}

MLEM program was used in this population modeling for each group. Mean and SD refer to population mean and population standard deviation; IIV refers to inter-individual variability as of $\% \mathrm{CV}$ 

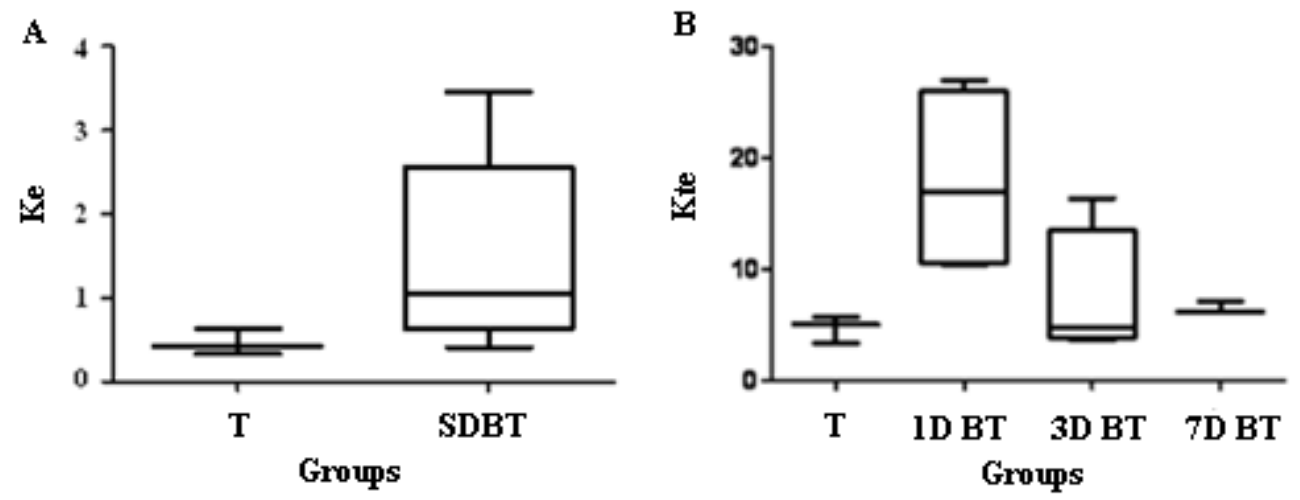

Figure 9. The effect of treatment regimen on Ke and Kte

A: Higher Ke associated with SDBT; B: Differential Kte associated with the timing of SDBT. 
variability for Kte decreased from $308 \%$ to $162 \%$. However, the pharmacokinetic analysis showed that the use of MDBT had no effect on the pharmacokinetics of TPT in plasma and tumor compartment.

The results of this analysis indicated that SDBT was associated with higher systemic elimination rate of TPT given after BEV compared to TPT given alone. In addition, the elimination rate of TPT from tumor compartment was dependent upon the schedule of BEV and TPT: increased after 1 day treatment of BEV and gradually decreased after 3 days and 7 days treatment of BEV. SDBT did produce a trend toward higher intratumoral exposure and penetration of TPT given 1 day after BEV administration compared to either TPT given alone or TPT given 3 days and 7 days after $\mathrm{BEV}$, however, the current experiment had a low power to identify the significant increase in intratumoral exposure and penetration of TPT after 1 day pre-treatment of BEV.

\subsubsection{The antitumor activity of the combination therapy}

In the SDBT groups, as shown in Figure 10, TPT significantly enhanced the antitumor activity of BEV when comparing final tumor volume between group B $v s$ groups 1D BT (mean \pm SD: $1525 \pm 322$ vs $1019 \pm 199, p<0.01$ ) and 7D BT (mean \pm SD: $1525 \pm 322$ vs $976 \pm 102, p<0.01)$. However, the pre-treatment of BEV did not enhance the antitumor activity of TPT when comparing group T with groups $1 \mathrm{D}$ TB $(p=0.108)$ and 7D BT $(p=0.129)$. Additionally, there is no significant difference in final tumor volume between groups 1D BT and 7D BT. In the MDBT groups, as shown in Figure 11, TPT had no effect the antitumor activity of multiple doses BEV when comparing final tumor volume between group $\mathrm{mB} v s$ groups $1 \mathrm{D} \mathrm{mBT}, 3 \mathrm{D} \mathrm{mBT}$ and $7 \mathrm{D} \mathrm{mBT}$. The treatment of multiple doses BEV had significant effect on the tumor growth delay when comparing group mT with groups $1 \mathrm{D} \mathrm{mBT}$ (mean $\pm \mathrm{SD}: 3210 \pm 670$ s $2380 \pm 493, p<0.01$ ), 3D mBT (mean \pm SD: $3210 \pm 670 v s 2104 \pm 378, p<0.01)$ and $7 \mathrm{D} \mathrm{mBT}(\operatorname{mean} \pm$ SD: $3210 \pm 670 v s$ $2366 \pm 660, p<0.05)$. And there is no significant difference in final tumor volume between groups 1D mBT, 3D mBT and 7D mBT. Thus, the effect on tumor growth after MDBT was likely due to the multiple doses BEV.

The results of this analysis indicated the antitumor activity of combination therapy was significantly enhanced after addition of TPT, but not after addition of BEV after SDBT. The antitumor activity of combination therapy and BEV monotherapy was equivalent and mainly due to the multiple doses of BEV in the MDBT groups. Additionally, the antitumor activity of TPT was not dependent on the schedule of BEV and TPT after either SDBT or MDBT. 


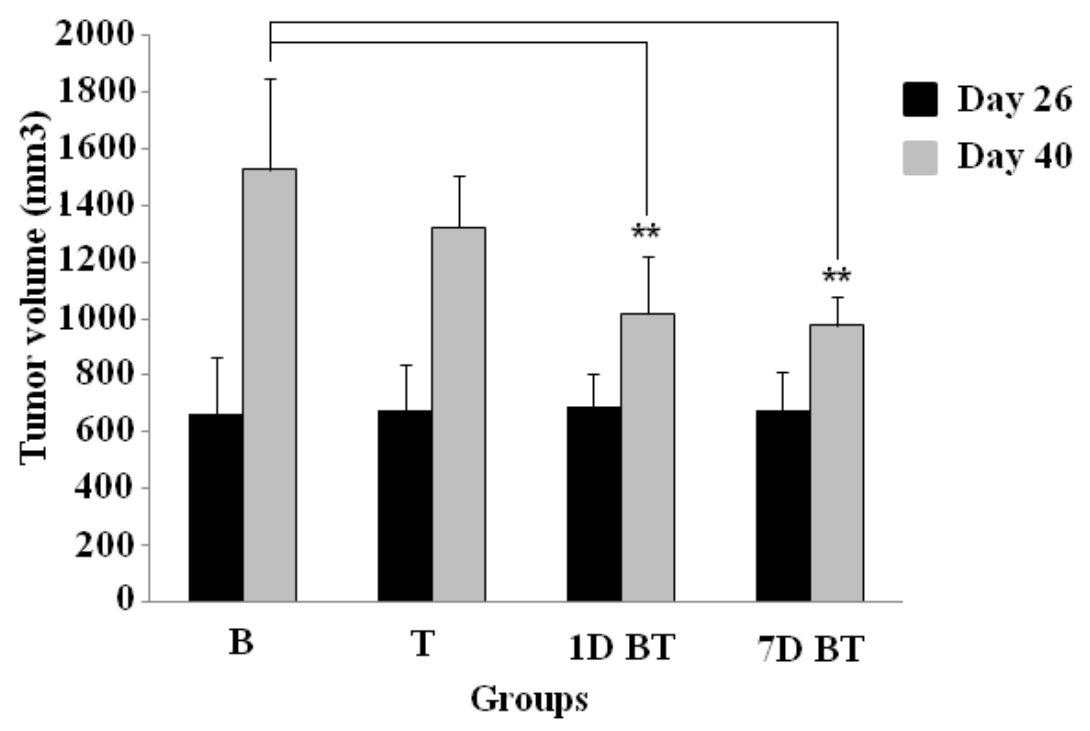

Figure 10. The effect of different schedule of SDBT combined with TPT on the growth of orthotopic Rh30 tumors.

Mice were size matched at day 26 (black columns) and tumor volume was measured after treatment at day 40 (gray columns). ${ }^{* *}, p=0.01$ comparing the final tumor volume in mice treated with TPT 1 day or 7 days after BEV with mice that received BEV only. 


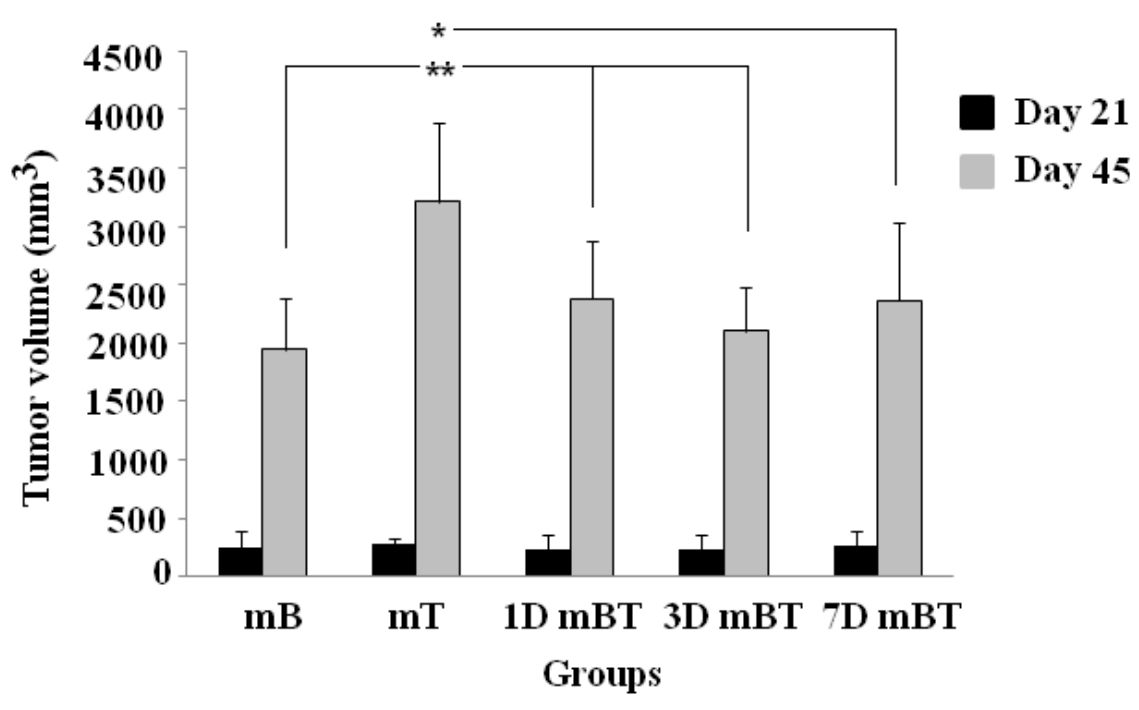

Figure 11. The effect of different schedule of MDBT combined with TPT on the growth of orthotopic Rh30 tumors.

Treatment was initiated when tumor volume reached approximately $200 \mathrm{~mm}^{3}$ at day 21 (black columns). Tumor volume was measured after treatment at day 45 (gray columns). **, $p=0.01$ comparing the final tumor volume in mice treated with BEV only with mice that received TPT only. ${ }^{* *}, p=0.01$ comparing the final tumor volume in mice treated with TPT 1 day or 3 days after BEV with mice that received TPT only. ${ }^{*}, p=0.05$ comparing the final tumor volume in mice treated with TPT 7 days after BEV with mice that received TPT only. 


\section{CHAPTER 3. DISCUSSION AND CONCLUSIONS}

The combination of angiogenesis inhibitors and cytotoxic drugs are currently investigated because of their different cellular targeting and non-overlapping toxicity of these two class drugs, and the encouraging response in preclinical studies and clinical trials $[49,51,154,182,183]$. However, the optimal scheduling and dosing of the combination is not well defined and has a pivotal role in the success of this regimen [14, 146]. Furthermore, the potential role of angiogenesis inhibitors in cytotoxic penetration and pharmacokinetic changes is also unclear and controversial [91, 147]. BEV is one of the better angiogenesis inhibitors due to its prevailing benefits in facilitating cytotoxic drugs treatment. TPT is ARMS-sensitive drug and currently used in treatment of RMS, however, as most cytotoxic drugs, clinical trials showed RMS is also resistant to TPT. One of the strategies to overcome drug resistance is to increase the intratumoral concentration and BEV administration has been proposed as a pharmacological means to normalize tumor vasculature and thereby enhance TPT penetration. This is the first study to determine the optimal scheduling of BEV and the cytotoxic drug TPT in the treatment of ARMS and to assess the effect of BEV on the pharmacokinetics and antitumor activity of TPT.

First, we conducted microdialysis studies to obtain total unbound TPT concentration in tumor ECF and plasma after TPT combined with a single dose or multiple doses of BEV at different schedule. We used a three compartmental PK model previously used in our lab to predict TPT concentration-time profiles in tumor ECF and plasma as well as PK parameters for individual mouse. However, the model poorly predicted the TPT concentration in tumor ECF with large -log likelihood. In addition, the PK parameters from this conventional model were always with huge variability. In order to better predict the concentration-time profile in tumor ECF and accurately describe the PK of TPT, we modified the conventional model. The modified PK model in Figure 4 significantly improved the model fit with a significant reduction of -log likelihood for each mouse and the variability of predicted PK parameters were within reasonable range $(\mathrm{CV} \%<40 \%)$ for individual mouse. Furthermore, in the three compartmental PK model CLin and CLout were used to describe the drug transfer between central and tumor compartment while only CLd was used to describe the drug transfer between central and peripheral compartment. The use of CLin and CLout were not justified and may be the cause of the huge variability of PK parameters. In the modified multi-compartmental PK model, all of the terms we used were the elimination rate constants and the model was more stable.

We observed an increase in TPT systemic elimination when TPT was administered after a single dose of BEV, and covariate analysis showed SDBT was associated with an increased systemic elimination rate $(\mathrm{Ke})$ of TPT. Meanwhile SDBT had no effect on the volume of distribution in central compartment (Vc) as shown in Table 4, resulting in an increase in the systemic clearance of TPT $(\mathrm{CL}=\mathrm{Vc} \times \mathrm{Ke})$. The mechanisms that underlie the changes in TPT systemic elimination and clearance after SDBT are unknown. It could be attributed to the altered renal clearance or the blocking of 
efflux transporters. TPT is a camptothecin and topoisomerase I inhibitor [184-186] and undergoes both renal and hepatic elimination [167, 187]. TPT is a substrate of efflux transporters such as P-glycoprotein (P-gp) and breast cancer resistance protein (BCRP) $[168,188]$. Although no publications have shown efflux transporters are blocked by macromolecular antiangiogenic agents, but only by small molecular angiogenesis inhibitors [189-192], a recent paper published this year [193] has indicated that there was a significant negative correlation between VEGF level and creatinine clearance. A decreasing VEGF level may increase creatinine clearance, especially for patients with impaired renal function that is associated with elevated angiogenic growth factors such as VEGF, VEGF receptors, Ang-1 and Ang-2. Thus SDBT may induce the renal clearance of TPT and affect the systemic elimination rate and clearance of TPT.

PK modeling also indicated that TPT elimination from the tumor compartment was significantly increased after 1 day of a single dose BEV treatment and gradually decreased to the control level after 3 days and 7 days of a single dose BEV administration. This observation supports the concept of vascular normalization proposed by Jain [14]. The vasculature in solid tumors is structurally and functionally abnormal[14] and lymphatic vessels also become dysfunctional, resulting in interstitial hypertension in solid tumors caused by lack of a drainage system [17, 22]. Consequently, cytotoxic drugs that have been delivered to the tumor compartment could be confined in the tumor and a reduction in elimination rate of the cytotoxic drugs from the tumor compartment is expected. Blocking angiogenesis leads to elimination of immature blood vessels and relief of the tumor microenvironment stress [50, 194]. Normalization of the lymphatic system was also documented by several studies [195, 196]. One of the possible mechanisms for the increased TPT tumor elimination after 1 day of BEV treatment could be the normalization of blood vessels and lymphatic system by BEV. The normalized, functional lymphatic vessels can drain out TPT from the tumor more rapidly. As the normalization effect of BEV diminishes over time, the lymphatic vessels lose their transport ability again and cytotoxic drugs are restricted in the tumor and have lower elimination rate. Additionally, the relieved interstitial hypertension could be another contributor to the increased TPT tumor elimination. Through the transient normalization effect of BEV, the decreased interstitial hypertension allows TPT to diffuse out from the tumor more rapidly. But when the interstitial hypertension increases again after the normalization effect of BEV disappears, the diffusion rate of TPT gradually decreases to control level.

In the microdialysis studies for TPT penetration after SDBT, we observed a trend in changes in TPT penetration. However, due to the low power of the experiment we did not identify which schedule of the combination treatment changed the TPT penetration. Thus in future studies, we may add more animals in each group to insure the experiment has sufficient power to determine whether the pre-treatment of BEV may enhance the penetration of TPT in Rh30 xenograft. Additionally, TPT penetration AUCt/AUCp was the overall display of the pharmacokinetics of TPT in tumor and plasma compartment. The results indicated that 1 day pre-treatment of BEV was associated with an increase in the systemic clearance of TPT, resulting from the increased Ke and on changes on Vc, leads to a decrease in AUCp. The covariate analysis showed that 1 day pre-treatment of 
$\mathrm{BEV}$ was associated with an increase in Kte and a decrease in $\mathrm{Vt}$, which may lead to an increase in AUCt. Thus, we may expect an increase in AUCt/AUCp ratio. Indeed, the absolute value of AUCt/AUCp was increased, but the increase did not reach a significant level. In the microdialysis studies for TPT penetration after MDBT, we didn't observe difference in TPT penetration. The exact underlying mechanism is unknown but could be due to the net balance of the anti-VEGF-induced pharmacodynamic effect on tumor vasculature and microenvironment changes [147]. Following anti-VEGF treatment, the tumor vasculature may undergo normalization with more functional blood vessels, increased blood flow and perfusion and decreased interstitial pressure. The entire tumor vasculature normalization effect following BEV administration would increase the tumor uptake of cytotoxic drugs [91]. However, these blood vessels' morphology and microenvironment changes are not the sole effect caused by anti-VEGF treatment. The anti-VEGF agent may also over prune the blood vessels and result in a substantial decrease in blood vessel surface area and blood vessel permeability, which decreased cytotoxic drug penetration into tumor tissue [146]. Thus after 1 day, 3 days or 7 days of the BEV administration, the tumor vasculature underwent these pharmacodynamic changes, and the overall effect on TPT penetration may cancel out each other-the increased intratumoral concentration by vasculature normalization is compensated by the deceased intratumoral concentration by over-pruning blood vessels. Moreover, for the multiple doses of BEV groups, another factor that may contribute the observed phenomenon is the anti-drug antibodies (ADA). Administration of therapeutic proteins can lead to unexpected immunogenicity in recipients of these products [197]. Repeated administration of BEV may induce the production of its ADA, and the ADA would interfere with the target of BEV as observed in the usage of an anti-angiogenesis inhibitor [198]. This would further result in a diminished effect on VEGF inhibition and the tumor vasculature normalization. This explains why we didn't see any changes in tumor exposure and penetration of TPT after MDBT compared to no BEV treatment.

Antitumor activity after a single dose of BEV and TPT combination therapy is superior to either agent used alone as shown in Figure 10. Currently, the prevailing rationale for the combination therapy of anti-angiogenic agents and cytotoxic drugs is anti-angiogenic agents induce tumor vasculature normalization and further increase cytotoxic drug penetration and efficacy $[14,49,154]$. However, enhanced antitumor activity can be achieved by combining the anti-angiogenic agents with cytotoxic drugs, despite a substance decrease in tumor uptake of the cytotoxic drugs [199, 200]. In our study, the additive effect on antitumor activity after combining BEV and TPT was observed, yet the penetration into tumor tissue and the tumor uptake of TPT did not alter or was even lower (T vs. 7D BT) after a single dose of BEV. As proposed by Dr. Waxman [91], overall anti-tumor activity is not solely determined by tumor cells' exposure to cytotoxic drugs. Rather, it is determined by the net balance between the angiogenesis inhibition-induced tumor cell starvation and the tumor cytotoxicity due to the exposure to cytotoxic drugs. Additionally, cytotoxic drugs may enhance the activity of anti-angiogenic agents and increase the sensitivity of blood vessels to VEGF inhibition, thereby augmenting antitumor activity in the combination setting [201], even with decreased cytotoxic drugs exposure in the tumor. Thus the increased antitumor activity for the combination therapy might derive from the above two reasons. 
Furthermore, although the volume of distribution in tumor compartment is not identical to tumor volume, it may serve as an early indicator of the therapeutic response. In our PK study, we used population modeling and covariate analysis to assess the effect of BEV on the volume of distribution of TPT in tumor compartment at the day of TPT administration. The results indicated that the use of BEV significantly decreased the volume of distribution in tumor compartment, which was confirmed by the tumor efficacy study in a different cohort of mice-a significant tumor volume reduction was observed when compared the combination therapy to monotherapy after two weeks of the initial treatment. Last, the combination therapy with a single dose of BEV and TPT did enhance the tumor response; however, the combination therapy with multiple doses of BEV and TPT did not benefit antitumor activity. TPT alone had the least antitumor activity, while multiple BEV only had similar antitumor activity when combing TPT. This result clearly indicated that multiple doses of BEV dominated the tumor response and the antitumor activity caused by a single dose of TPT was diminished.

In summary, in the present study we observed significant changes in TPT disposition after a single BEV dose in our orthotopic RMS model. In brief, a single dose of BEV had significant effect on the systemic elimination and clearance of TPT, and time-dependently regulated TPT elimination from tumor compartment. Though a substantial antitumor activity after a single dose of BEV and TPT combination was detected, BEV did not alter TPT penetration or exposure into tumor tissue. Furthermore, TPT penetration and efficacy were not affected after multiple doses of BEV administration. Although using drug penetration and antitumor activity as the end points of the study, we were unable to identify the optimal schedule of the combination therapy. However, our results provide crucial insights into the effect of coadministration of BEV on TPT PK changes. The overall effects of anti-angiogenic agents on tumor vasculature and microenvironment, resulting in the net balance of the antiangiogenesis-mediated pharmacologic actions may be the determinants for the cytotoxic drug penetration. Cytotoxic drugs may reversely sensitize the antitumor activity of the anti-angiogenic agents. This study highlights the complexity of PKPD interaction that may take place when antiangiogenic agents and cytotoxic drugs are combined and cautions that careful consideration and more mechanistic investigation should be made before the usage of the combination of anti-angiogenic agents with cytotoxic drugs for cancer treatment. 


\section{LIST OF REFERENCES}

1. Fukumura, D. and R.K. Jain, Tumor microenvironment abnormalities: causes, consequences, and strategies to normalize. J Cell Biochem, 2007. 101(4): p. 937-49.

2. Tredan, O., et al., Drug resistance and the solid tumor microenvironment. J Natl Cancer Inst, 2007. 99(19): p. 1441-54.

3. Vaupel, P., Tumor microenvironmental physiology and its implications for radiation oncology. Semin Radiat Oncol, 2004. 14(3): p. 198-206.

4. Zhu, Z., Aberrant tumor vasculature and angiogenesis: new opportunities for cancer therapy. Drug Discov Today, 2003. 8(18): p. 827-8.

5. Vaupel, P.W., The influence of tumor blood flow and microenvironmental factors on the efficacy of radiation, drugs and localized hyperthermia. Klin Padiatr, 1997. 209(4): p. 243-9.

6. Lockman, P.R., et al., Heterogeneous blood-tumor barrier permeability determines drug efficacy in experimental brain metastases of breast cancer. Clin Cancer Res, 2011. 16(23): p. 5664-78.

7. Lutsenko, S.V., S.M. Kiselev, and S.E. Severin, Molecular mechanisms of tumor angiogenesis. Biochemistry (Mosc), 2003. 68(3): p. 286-300.

8. Sato, Y., Molecular diagnosis of tumor angiogenesis and anti-angiogenic cancer therapy. Int J Clin Oncol, 2003. 8(4): p. 200-6.

9. Weis, S.M. and D.A. Cheresh, Tumor angiogenesis: molecular pathways and therapeutic targets. Nat Med, 2011. 17(11): p. 1359-70.

10. Jain, R.K., Determinants of tumor blood flow: a review. Cancer Res, 1988. 48(10): p. 2641-58.

11. Benjamin, L.E., et al., Selective ablation of immature blood vessels in established human tumors follows vascular endothelial growth factor withdrawal. J Clin Invest, 1999. 103(2): p. 159-65.

12. Hashizume, H., et al., Openings between defective endothelial cells explain tumor vessel leakiness. Am J Pathol, 2000. 156(4): p. 1363-80.

13. Aref, M., M. Brechbiel, and E.C. Wiener, Identifying tumor vascular permeability heterogeneity with magnetic resonance imaging contrast agents. Invest Radiol, 2002. 37(4): p. 178-92.

14. Jain, R.K., Normalization of tumor vasculature: an emerging concept in antiangiogenic therapy. Science, 2005. 307(5706): p. 58-62.

15. Sevick, E.M. and R.K. Jain, Geometric resistance to blood flow in solid tumors perfused ex vivo: effects of tumor size and perfusion pressure. Cancer Res, 1989. 49(13): p. 3506-12.

16. Sevick, E.M. and R.K. Jain, Effect of red blood cell rigidity on tumor blood flow: increase in viscous resistance during hyperglycemia. Cancer Res, 1991. 51(10): p. 2727-30.

17. Padera, T.P., et al., Pathology: cancer cells compress intratumour vessels. Nature, 2004. 427(6976): p. 695.

18. Gillies, R.J., et al., Causes and effects of heterogeneous perfusion in tumors. Neoplasia, 1999. 1(3): p. 197-207. 
19. Sleeman, J., A. Schmid, and W. Thiele, Tumor lymphatics. Semin Cancer Biol, 2009. 19(5): p. 285-97.

20. Boucher, Y., M. Leunig, and R.K. Jain, Tumor angiogenesis and interstitial hypertension. Cancer Res, 1996. 56(18): p. 4264-6.

21. Stracke, M.L., et al., The role of the extracellular matrix in tumor cell metastasis. In Vivo, 1994. 8(1): p. 49-58.

22. Boucher, Y., L.T. Baxter, and R.K. Jain, Interstitial pressure gradients in tissue-isolated and subcutaneous tumors: implications for therapy. Cancer Res, 1990. 50(15): p. 4478-84.

23. Jain, R.K. and L.T. Baxter, Mechanisms of heterogeneous distribution of monoclonal antibodies and other macromolecules in tumors: significance of elevated interstitial pressure. Cancer Res, 1988. 48(24 Pt 1): p. 7022-32.

24. Rintoul, R.C. and T. Sethi, The role of extracellular matrix in small-cell lung cancer. Lancet Oncol, 2001. 2(7): p. 437-42.

25. Netti, P.A., et al., Role of extracellular matrix assembly in interstitial transport in solid tumors. Cancer Res, 2000. 60(9): p. 2497-503.

26. Lochter, A. and M.J. Bissell, Involvement of extracellular matrix constituents in breast cancer. Semin Cancer Biol, 1995. 6(3): p. 165-73.

27. Stewart, D.A., C.R. Cooper, and R.A. Sikes, Changes in extracellular matrix (ECM) and ECM-associated proteins in the metastatic progression of prostate cancer. Reprod Biol Endocrinol, 2004. 2: p. 2.

28. Davies Cde, L., et al., Comparison of IgG diffusion and extracellular matrix composition in rhabdomyosarcomas grown in mice versus in vitro as spheroids reveals the role of host stromal cells. Br J Cancer, 2002. 86(10): p. 1639-44.

29. Brown, E., et al., Dynamic imaging of collagen and its modulation in tumors in vivo using second-harmonic generation. Nat Med, 2003. 9(6): p. 796-800.

30. Harris, A.L., Hypoxia--a key regulatory factor in tumour growth. Nat Rev Cancer, 2002. 2(1): p. 38-47.

31. Liao, D. and R.S. Johnson, Hypoxia: a key regulator of angiogenesis in cancer. Cancer Metastasis Rev, 2007. 26(2): p. 281-90.

32. Fukamachi, T., et al., Tumor specific low pH environments enhance the cytotoxicity of lovastatin and cantharidin. Cancer Lett, 2011. 297(2): p. 182-9.

33. Brown, J.M., Tumor microenvironment and the response to anticancer therapy. Cancer Biol Ther, 2002. 1(5): p. 453-8.

34. Thomlinson, R.H. and L.H. Gray, The histological structure of some human lung cancers and the possible implications for radiotherapy. Br J Cancer, 1955. 9(4): p. 539-49.

35. Brown, J.M. and A.J. Giaccia, The unique physiology of solid tumors: opportunities (and problems) for cancer therapy. Cancer Res, 1998. 58(7): p. 1408-16.

36. Helmlinger, G., et al., Acid production in glycolysis-impaired tumors provides new insights into tumor metabolism. Clin Cancer Res, 2002. 8(4): p. 1284-91.

37. Jain, R.K., Delivery of molecular and cellular medicine to solid tumors. Adv Drug Deliv Rev, 2001. 46(1-3): p. 149-68. 
38. Kim, S.H., H.J. Kuh, and C.R. Dass, The reciprocal interaction: chemotherapy and tumor microenvironment. Curr Drug Discov Technol, 2011. 8(2): p. 102-6.

39. Less, J.R., et al., Microvascular architecture in a mammary carcinoma: branching patterns and vessel dimensions. Cancer Res, 1991. 51(1): p. 265-73.

40. Yuan, F., et al., Vascular permeability and microcirculation of gliomas and mammary carcinomas transplanted in rat and mouse cranial windows. Cancer Res, 1994. 54(17): p. 4564-8.

41. Durand, R.E., Intermittent blood flow in solid tumours--an under-appreciated source of 'drug resistance'. Cancer Metastasis Rev, 2001. 20(1-2): p. 57-61.

42. Jain, R.K. and T. Stylianopoulos, Delivering nanomedicine to solid tumors. Nat Rev Clin Oncol, 2010. 7(11): p. 653-64.

43. Baxter, L.T. and R.K. Jain, Transport of fluid and macromolecules in tumors. I. Role of interstitial pressure and convection. Microvasc Res, 1989. 37(1): $\mathrm{p}$. 77-104.

44. Grantab, R., S. Sivananthan, and I.F. Tannock, The penetration of anticancer drugs through tumor tissue as a function of cellular adhesion and packing density of tumor cells. Cancer Res, 2006. 66(2): p. 1033-9.

45. Lalani, A.S., et al., Selective tumor targeting by the hypoxia-activated prodrug AQ4N blocks tumor growth and metastasis in preclinical models of pancreatic cancer. Clin Cancer Res, 2007. 13(7): p. 2216-25.

46. Cowan, D.S. and I.F. Tannock, Factors that influence the penetration of methotrexate through solid tissue. Int J Cancer, 2001. 91(1): p. 120-5.

47. Lee, C.M. and I.F. Tannock, Inhibition of endosomal sequestration of basic anticancer drugs: influence on cytotoxicity and tissue penetration. $\mathrm{Br} \mathrm{J}$ Cancer, 2006. 94(6): p. 863-9.

48. Gerber, H.P. and N. Ferrara, Pharmacology and pharmacodynamics of bevacizumab as monotherapy or in combination with cytotoxic therapy in preclinical studies. Cancer Res, 2005. 65(3): p. 671-80.

49. Yanagisawa, M., et al., Bevacizumab improves the delivery and efficacy of paclitaxel. Anticancer Drugs, 2010. 21(7): p. 687-94.

50. Tong, R.T., et al., Vascular normalization by vascular endothelial growth factor receptor 2 blockade induces a pressure gradient across the vasculature and improves drug penetration in tumors. Cancer Res, 2004. 64(11): p. 3731-6.

51. Zhou, Q., P. Guo, and J.M. Gallo, Impact of angiogenesis inhibition by sunitinib on tumor distribution of temozolomide. Clin Cancer Res, 2008. 14(5): p. 1540-9.

52. Eikenes, L., et al., Collagenase increases the transcapillary pressure gradient and improves the uptake and distribution of monoclonal antibodies in human osteosarcoma xenografts. Cancer Res, 2004. 64(14): p. 4768-73.

53. Jang, S.H., M.G. Wientjes, and J.L. Au, Enhancement of paclitaxel delivery to solid tumors by apoptosis-inducing pretreatment: effect of treatment schedule. $\mathrm{J}$ Pharmacol Exp Ther, 2001. 296(3): p. 1035-42.

54. Kaasgaard, T. and T.L. Andresen, Liposomal cancer therapy: exploiting tumor characteristics. Expert Opin Drug Deliv, 2010. 7(2): p. 225-43.

55. Wang, M. and M. Thanou, Targeting nanoparticles to cancer. Pharmacol Res, 2010. 62(2): p. 90-9. 
56. ElBayoumi, T.A. and V.P. Torchilin, Tumor-targeted nanomedicines: enhanced antitumor efficacy in vivo of doxorubicin-loaded, long-circulating liposomes modified with cancer-specific monoclonal antibody. Clin Cancer Res, 2009. 15(6): p. 1973-80.

57. Gupta, Y., et al., Design and development of folate appended liposomes for enhanced delivery of 5-FU to tumor cells. J Drug Target, 2007. 15(3): p. 231-40.

58. Eavarone, D.A., X. Yu, and R.V. Bellamkonda, Targeted drug delivery to C6 glioma by transferrin-coupled liposomes. J Biomed Mater Res, 2000. 51(1): p. 10-4.

59. Murphy, E.A., et al., Nanoparticle-mediated drug delivery to tumor vasculature suppresses metastasis. Proc Natl Acad Sci U S A, 2008. 105(27): p. 9343-8.

60. Gardlik, R. and J.H. Fruehauf, Bacterial vectors and delivery systems in cancer therapy. IDrugs, 2010. 13(10): p. 701-6.

61. de Castro Junior, G., et al., Angiogenesis and cancer: A cross-talk between basic science and clinical trials (the "do ut des" paradigm). Crit Rev Oncol Hematol, 2006. 59(1): p. 40-50.

62. Minchinton, A.I. and I.F. Tannock, Drug penetration in solid tumours. Nat Rev Cancer, 2006. 6(8): p. 583-92.

63. Bid, H.K. and P.J. Houghton, Targeting angiogenesis in childhood sarcomas. Sarcoma, 2011. 2011: p. 601514.

64. Folkman, J., How is blood vessel growth regulated in normal and neoplastic tissue? G.H.A. Clowes memorial Award lecture. Cancer Res, 1986. 46(2): p. 467-73.

65. Ferrara, N. and R.S. Kerbel, Angiogenesis as a therapeutic target. Nature, 2005. 438(7070): p. 967-74.

66. Ferrara, N., et al., Heterozygous embryonic lethality induced by targeted inactivation of the VEGF gene. Nature, 1996. 380(6573): p. 439-42.

67. Suzuki, S., et al., Clinicopathological significance of platelet-derived growth factor (PDGF)-B and vascular endothelial growth factor-A expression, PDGF receptor-beta phosphorylation, and microvessel density in gastric cancer. BMC Cancer, 2010. 10: p. 659.

68. Mahalingam, D., et al., Targeting sarcomas: novel biological agents and future perspectives. Curr Drug Targets, 2009. 10(10): p. 937-49.

69. Zhang, J., et al., Elevated expression of vascular endothelial growth factor correlates with increased angiogenesis and decreased progression-free survival among patients with low-grade neuroendocrine tumors. Cancer, 2007. 109(8): p. 1478-86.

70. Kowanetz, M. and N. Ferrara, Vascular endothelial growth factor signaling pathways: therapeutic perspective. Clin Cancer Res, 2006. 12(17): p. 5018-22.

71. Olsson, A.K., et al., VEGF receptor signalling - in control of vascular function. Nat Rev Mol Cell Biol, 2006. 7(5): p. 359-71.

72. Kim, K.J., et al., Inhibition of vascular endothelial growth factor-induced angiogenesis suppresses tumour growth in vivo. Nature, 1993. 362(6423): p. 841-4.

73. Folkman, J., Tumor angiogenesis: therapeutic implications. N Engl J Med, 1971. 285(21): p. 1182-6. 
74. Taylor, S. and J. Folkman, Protamine is an inhibitor of angiogenesis. Nature, 1982. 297(5864): p. 307-12.

75. Crum, R., S. Szabo, and J. Folkman, A new class of steroids inhibits angiogenesis in the presence of heparin or a heparin fragment. Science, 1985. 230(4732): p. 1375-8.

76. Maeshima, Y., et al., Tumstatin, an endothelial cell-specific inhibitor of protein synthesis. Science, 2002. 295(5552): p. 140-3.

77. O'Reilly, M.S., et al., Angiostatin: a novel angiogenesis inhibitor that mediates the suppression of metastases by a Lewis lung carcinoma. Cell, 1994. 79(2): p. 315-28.

78. Frater-Schroder, M., et al., Tumor necrosis factor type alpha, a potent inhibitor of endothelial cell growth in vitro, is angiogenic in vivo. Proc Natl Acad Sci U S A, 1987. 84(15): p. 5277-81.

79. Abdollahi, A., et al., Endostatin's antiangiogenic signaling network. Mol Cell, 2004. 13(5): p. 649-63.

80. Hurwitz, H., et al., Bevacizumab plus irinotecan, fluorouracil, and leucovorin for metastatic colorectal cancer. N Engl J Med, 2004. 350(23): p. 2335-42.

81. McGee, M.C., et al., Improved intratumoral oxygenation through vascular normalization increases glioma sensitivity to ionizing radiation. Int J Radiat Oncol Biol Phys, 2010. 76(5): p. 1537-45.

82. Finn, R.S., et al., Targeting vascular endothelial growth factor with the monoclonal antibody bevacizumab inhibits human hepatocellular carcinoma cells growing in an orthotopic mouse model. Liver Int, 2009. 29(2): p. 284-90.

83. Okada, Y., et al., The effect of bevacizumab on tumour growth of malignant fibrous histiocytoma in an animal model. Anticancer Res, 2010. 30(9): p. 3391-5.

84. Tol, J., et al., Chemotherapy, bevacizumab, and cetuximab in metastatic colorectal cancer. N Engl J Med, 2009. 360(6): p. 563-72.

85. Amselem, L., et al., Intravitreal bevacizumab (Avastin) for choroidal metastasis secondary to breast carcinoma: short-term follow-up. Eye (Lond), 2007. 21(4): p. 566-7.

86. de Gramont, A. and E. Van Cutsem, Investigating the potential of bevacizumab in other indications: metastatic renal cell, non-small cell lung, pancreatic and breast cancer. Oncology, 2005. 69 (Suppl 3): p. 46-56.

87. Uegami, S., et al., A case of synchronous multiple liver metastasis of sigmoid colon cancer with a pathological complete response to combination chemotherapy of 5-FU/LV and bevacizumab. Gan To Kagaku Ryoho, 2011. 38(2): p. 321-4.

88. Nishida, K., et al., A case with liver resection of metastasis from rectal cancer after bevacizumab treatment. Gan To Kagaku Ryoho, 2010. 37(12): p. 2517-9.

89. Imaizumi, T., et al., Suppressive effect of bevacizumab on peritoneal dissemination from gastric cancer in a peritoneal metastasis model. Surg Today, 2010. 40(9): p. 851-7.

90. Yang, H., M.J. Jager, and H.E. Grossniklaus, Bevacizumab suppression of establishment of micrometastases in experimental ocular melanoma. Invest Ophthalmol Vis Sci, 2010. 51(6): p. 2835-42. 
91. Ma, J. and D.J. Waxman, Combination of antiangiogenesis with chemotherapy for more effective cancer treatment. Mol Cancer Ther, 2008. 7(12): p. 3670-84.

92. Bagri, A., et al., Use of anti-VEGF adjuvant therapy in cancer: challenges and rationale. Trends Mol Med, 2010. 16(3): p. 122-32.

93. Hicklin, D.J. and L.M. Ellis, Role of the vascular endothelial growth factor pathway in tumor growth and angiogenesis. J Clin Oncol, 2005. 23(5): p. 1011-27.

94. Kerbel, R.S., Antiangiogenic therapy: a universal chemosensitization strategy for cancer? Science, 2006. 312(5777): p. 1171-5.

95. Teicher, B.A., A systems approach to cancer therapy. (Antioncogenics + standard cytotoxics-->mechanism(s) of interaction). Cancer Metastasis Rev, 1996. 15(2): p. 247-72.

96. Miller, K., et al., Paclitaxel plus bevacizumab versus paclitaxel alone for metastatic breast cancer. N Engl J Med, 2007. 357(26): p. 2666-76.

97. Cao, Y., et al., A meta-analysis of randomized controlled trials comparing chemotherapy plus bevacizumab with chemotherapy alone in metastatic colorectal cancer. Int J Colorectal Dis, 2009. 24(6): p. 677-85.

98. Kabbinavar, F., et al., Phase II, randomized trial comparing bevacizumab plus fluorouracil (FU)/leucovorin ( $L V$ ) with FU/LV alone in patients with metastatic colorectal cancer. J Clin Oncol, 2003. 21(1): p. 60-5.

99. Sandler, A., et al., Paclitaxel-carboplatin alone or with bevacizumab for non-small-cell lung cancer. N Engl J Med, 2006. 355(24): p. 2542-50.

100. Herbst, R.S., et al., Phase II study of efficacy and safety of bevacizumab in combination with chemotherapy or erlotinib compared with chemotherapy alone for treatment of recurrent or refractory non small-cell lung cancer. J Clin Oncol, 2007. 25(30): p. 4743-50.

101. Johnson, D.H., et al., Randomized phase II trial comparing bevacizumab plus carboplatin and paclitaxel with carboplatin and paclitaxel alone in previously untreated locally advanced or metastatic non-small-cell lung cancer. J Clin Oncol, 2004. 22(11): p. 2184-91.

102. Hayes-Jordan, A. and R. Andrassy, Rhabdomyosarcoma in children. Curr Opin Pediatr, 2009. 21(3): p. 373-8.

103. Paulino, A.C. and M.F. Okcu, Rhabdomyosarcoma. Curr Probl Cancer, 2008. 32(1): p. 7-34.

104. Parham, D.M., Pathologic classification of rhabdomyosarcomas and correlations with molecular studies. Mod Pathol, 2001. 14(5): p. 506-14.

105. Beverly Raney, R., et al., Results of the Intergroup Rhabdomyosarcoma Study Group D9602 protocol, using vincristine and dactinomycin with or without cyclophosphamide and radiation therapy, for newly diagnosed patients with low-risk embryonal rhabdomyosarcoma: a report from the Soft Tissue Sarcoma Committee of the Children's Oncology Group. J Clin Oncol, 2011. 29(10): p. 1312-8.

106. Chisholm, J.C., et al., Prognostic factors after relapse in nonmetastatic rhabdomyosarcoma: a nomogram to better define patients who can be salvaged with further therapy. J Clin Oncol, 2011. 29(10): p. 1319-25. 
107. Perez, E.A., et al., Rhabdomyosarcoma in Children: A SEER Population Based Study. J Surg Res, 2011. 170(2): p. 243-51.

108. Riopelle, J.L. and J.P. Theriault, An unknown type of soft part sarcoma: alveolar rhabdomyosarcoma. Ann Anat Pathol (Paris), 1956. 1(1): p. 88-111.

109. Mazzoleni, S., et al., Outcomes and prognostic factors after recurrence in children and adolescents with nonmetastatic rhabdomyosarcoma. Cancer, 2005. 104(1): p. 183-90.

110. Sultan, I., et al., Comparing adult and pediatric rhabdomyosarcoma in the surveillance, epidemiology and end results program, 1973 to 2005: an analysis of 2,600 patients. J Clin Oncol, 2009. 27(20): p. 3391-7.

111. Ognjanovic, S., et al., Trends in childhood rhabdomyosarcoma incidence and survival in the United States, 1975-2005. Cancer, 2009. 115(18): p. 4218-26.

112. Vassal, G., et al., Phase II trial of irinotecan in children with relapsed or refractory rhabdomyosarcoma: a joint study of the French Society of Pediatric Oncology and the United Kingdom Children's Cancer Study Group. J Clin Oncol, 2007. 25(4): p. 356-61.

113. Rodriguez-Perales, S., et al., Molecular cytogenetic characterization of rhabdomyosarcoma cell lines. Cancer Genet Cytogenet, 2004. 148(1): p. 35-43.

114. Felix, C.A., et al., Frequency and diversity of p53 mutations in childhood rhabdomyosarcoma. Cancer Res, 1992. 52(8): p. 2243-7.

115. Onisto, M., et al., Expression and activity of vascular endothelial growth factor and metalloproteinases in alveolar and embryonal rhabdomyosarcoma cell lines. Int J Oncol, 2005. 27(3): p. 791-8.

116. Zeng, F.Y., et al., PAX3-FKHR sensitizes human alveolar rhabdomyosarcoma cells to camptothecin-mediated growth inhibition and apoptosis. Cancer Lett, 2009. 284(2): p. 157-64.

117. Pawlik, C.A., et al., Effective schedules of exposure of medulloblastoma and rhabdomyosarcoma xenografts to topotecan correlate with in vitro assays. Clin Cancer Res, 1998. 4(8): p. 1995-2002.

118. Friedman, H.S., et al., Elevated DNA polymerase alpha, DNA polymerase beta, and DNA topoisomerase II in a melphalan-resistant rhabdomyosarcoma xenograft that is cross-resistant to nitrosoureas and topotecan. Cancer Res, 1994. 54(13): p. 3487-93.

119. Perez Martinez, A., et al., [Topotecan for pediatric patients with resistant and recurrent solid tumors]. An Pediatr (Barc), 2003. 59(2): p. 143-8.

120. Chu, J. and J.M. Gallo, Application of microdialysis to characterize drug disposition in tumors. Adv Drug Deliv Rev, 2000. 45(2-3): p. 243-53.

121. Mellett, L.B., Pharmacodynamic and pharmacokinetic measurements of antitumor agents. Clin Pharmacol Ther, 1974. 16(1): p. 230-42.

122. Sufrin, G. and P. Murphy, Pharmacokinetic studies in the chemotherapy of neuroblastoma using the C1300 murine system. Oncology, 1976. 33(4): p. 173-8.

123. Martini, A., et al., Antineoplastic activity and pharmacokinetics of adriamycin and daunomycin in tumor bearing mice. Oncology, 1977. 34(4): p. 173-8.

124. Denton, C.L. and D.L. Gustafson, Pharmacokinetics and pharmacodynamics of AZD6244 (ARRY-142886) in tumor-bearing nude mice. Cancer Chemother Pharmacol, 2011. 67(2): p. 349-60. 
125. Hao, M., et al., Pharmacokinetics and tissue distribution of 25-hydroxyprotopanaxadiol, an anti-cancer compound isolated from Panax ginseng, in athymic mice bearing xenografts of human pancreatic tumors. Eur J Drug Metab Pharmacokinet, 2011. 35(3-4): p. 109-13.

126. Chang, C.H., et al., Comparative evaluation of FET and FDG for differentiating lung carcinoma from inflammation in mice. Anticancer Res, 2006. 26(2A): p. 917-25.

127. Denardo, S.J., et al., 111In-LLP2A-DOTA Polyethylene Glycol-Targeting \{alpha\}4\{beta\}1 Integrin: Comparative Pharmacokinetics for Imaging and Therapy of Lymphoid Malignancies. J Nucl Med, 2009. 50(4): p. 625-34.

128. Shapiro, W.R., et al., Pharmacokinetics of tumor cell exposure to [14C] methotrexate after intracarotid administration without and with hyperosmotic opening of the blood-brain and blood-tumor barriers in rat brain tumors: a quantitative autoradiographic study. Cancer Res, 1988. 48(3): p. 694-701.

129. Hebert, E.M., et al., Preferential tumour accumulation of gold nanoparticles, visualised by Magnetic Resonance Imaging: radiosensitisation studies in vivo and in vitro. Int J Radiat Biol, 2010. 86(8): p. 692-700.

130. Jynge, P., et al., In vivo tissue pharmacokinetics by fluorine magnetic resonance spectroscopy: a study of liver and muscle disposition of fleroxacin in humans. Clin Pharmacol Ther, 1990. 48(5): p. 481-9.

131. de Lange, E.C., et al., Methodological considerations of intracerebral microdialysis in pharmacokinetic studies on drug transport across the blood-brain barrier. Brain Res Brain Res Rev, 1997. 25(1): p. 27-49.

132. Wolf, W., V. Waluch, and C.A. Presant, Non-invasive 19F-NMRS of 5-fluorouracil in pharmacokinetics and pharmacodynamic studies. NMR Biomed, 1998. 11(7): p. 380-7.

133. Joukhadar, C. and M. Muller, Microdialysis: current applications in clinical pharmacokinetic studies and its potential role in the future. Clin Pharmacokinet, 2005. 44(9): p. 895-913.

134. Delgado, J.M., et al., Dialytrode for long term intracerebral perfusion in awake monkeys. Arch Int Pharmacodyn Ther, 1972. 198(1): p. 9-21.

135. Manda, P., et al., Delivery of cefotaxime to the brain via intranasal administration. Drug Dev Ind Pharm, 2011. 37(11): p. 1306-10.

136. Zhang, Y.J., et al., Pharmacokinetics of phenolic compounds of Danshen extract in rat blood and brain by microdialysis sampling. J Ethnopharmacol, 2011. 136(1): p. 129-36.

137. Tsai, Y.J., L.C. Lin, and T.H. Tsai, Pharmacokinetics of adenosine and cordycepin, a bioactive constituent of Cordyceps sinensis in rat. J Agric Food Chem, 2010. 58(8): p. 4638-43.

138. Shukla, C., et al., Quantification and prediction of skin pharmacokinetics of amoxicillin and cefuroxime. Biopharm Drug Dispos, 2009. 30(6): p. 281-93.

139. Dukic, S., et al., Determination of free extracellular levels of methotrexate by microdialysis in muscle and solid tumor of the rabbit. Pharm Res, 1998. 15(1): p. $133-8$. 
140. Yu, W., et al., Microdialysis for pharmacokinetic-pharmacodynamic studies. Pharmazie, 2007. 62(12): p. 883-91.

141. Wei, Y.H., et al., Microdialysis: a technique for pharmacokinetic-pharmacodynamic studies of oncological drugs. Curr Pharm Biotechnol, 2009. 10(6): p. 631-40.

142. Zheng, H., et al., Determination of oxymatrine and its metabolite matrine in rat blood and dermal microdialysates by high throughput liquid chromatography/tandem mass spectrometry. J Pharm Biomed Anal, 2009. 49(2): p. 427-33.

143. Chang, Y.L., et al., Simultaneous determination of nicotine and its metabolite, cotinine, in rat blood and brain tissue using microdialysis coupled with liquid chromatography: pharmacokinetic application. J Chromatogr A, 2005. 1088(1-2): p. $152-7$.

144. Benveniste, H., et al., Regional cerebral glucose phosphorylation and blood flow after insertion of a microdialysis fiber through the dorsal hippocampus in the rat. J Neurochem, 1987. 49(3): p. 729-34.

145. Devineni, D., A. Klein-Szanto, and J.M. Gallo, Uptake of temozolomide in a rat glioma model in the presence and absence of the angiogenesis inhibitor TNP-470. Cancer Res, 1996. 56(9): p. 1983-7.

146. Ma, J., et al., Pharmacodynamic-mediated reduction of temozolomide tumor concentrations by the angiogenesis inhibitor TNP-470. Cancer Res, 2001. 61(14): p. 5491-8.

147. Ma, J., et al., Pharmacodynamic-mediated effects of the angiogenesis inhibitor SU5416 on the tumor disposition of temozolomide in subcutaneous and intracerebral glioma xenograft models. J Pharmacol Exp Ther, 2003. 305(3): p. 833-9.

148. Zhou, Q. and J.M. Gallo, Differential effect of sunitinib on the distribution of temozolomide in an orthotopic glioma model. Neuro Oncol, 2009. 11(3): p. 301-10.

149. Wildiers, H., et al., Effect of antivascular endothelial growth factor treatment on the intratumoral uptake of CPT-11. Br J Cancer, 2003. 88(12): p. 1979-86.

150. Franco, M., et al., Targeted anti-vascular endothelial growth factor receptor-2 therapy leads to short-term and long-term impairment of vascular function and increase in tumor hypoxia. Cancer Res, 2006. 66(7): p. 3639-48.

151. Dings, R.P., et al., Scheduling of radiation with angiogenesis inhibitors anginex and Avastin improves therapeutic outcome via vessel normalization. Clin Cancer Res, 2007. 13(11): p. 3395-402.

152. Denlinger, C.S., et al., Pharmacokinetic analysis of irinotecan plus bevacizumab in patients with advanced solid tumors. Cancer Chemother Pharmacol, 2009. 65(1): p. 97-105.

153. Horita, Y., et al., Effects of bevacizumab on plasma concentration of irinotecan and its metabolites in advanced colorectal cancer patients receiving FOLFIRI with bevacizumab as second-line chemotherapy. Cancer Chemother Pharmacol, 2010. 65(3): p. 467-71. 
154. Dickson, P.V., et al., Bevacizumab-induced transient remodeling of the vasculature in neuroblastoma xenografts results in improved delivery and efficacy of systemically administered chemotherapy. Clin Cancer Res, 2007. 13(13): p. 3942-50.

155. Myers, A.L., et al., Bevacizumab-induced tumor vessel remodeling in rhabdomyosarcoma xenografts increases the effectiveness of adjuvant ionizing radiation. J Pediatr Surg, 2010. 45(6): p. 1080-5.

156. Evans, N.D., et al., A mathematical model for the in vitro kinetics of the anti-cancer agent topotecan. Math Biosci, 2004. 189(2): p. 185-217.

157. Cheung, S.Y., et al., Exploration of the intercellular heterogeneity of topotecan uptake into human breast cancer cells through compartmental modelling. Math Biosci, 2008. 213(2): p. 119-34.

158. Chen, J., Q. Lu, and J.P. Balthasar, Mathematical modeling of topotecan pharmacokinetics and toxicodynamics in mice. J Pharmacokinet Pharmacodyn, 2007. 34(6): p. 829-47.

159. Blaney, S.M., et al., Plasma and cerebrospinal fluid pharmacokinetic study of topotecan in nonhuman primates. Cancer Res, 1993. 53(4): p. 725-7.

160. Shah, D.K. and J.P. Balthasar, Physiologically based pharmacokinetic model for topotecan in mice. J Pharmacokinet Pharmacodyn, 2011. 38(1): p. 121-42.

161. Guichard, S., et al., Schedule-dependent activity of topotecan in OVCAR-3 ovarian carcinoma xenograft: pharmacokinetic and pharmacodynamic evaluation. Clin Cancer Res, 2001. 7(10): p. 3222-8.

162. Freeman, B.B., 3rd, et al., Using plasma topotecan pharmacokinetics to estimate topotecan exposure in cerebrospinal fluid of children with medulloblastoma. Neuro Oncol, 2006. 8(2): p. 89-95.

163. Schaiquevich, P., et al., Population pharmacokinetic analysis of topotecan in pediatric cancer patients. Clin Cancer Res, 2007. 13(22 Pt 1): p. 6703-11.

164. Panetta, J.C., et al., Using pharmacokinetic and pharmacodynamic modeling and simulation to evaluate importance of schedule in topotecan therapy for pediatric neuroblastoma. Clin Cancer Res, 2008. 14(1): p. 318-25.

165. Baker, S.D., et al., Cerebrospinal fluid pharmacokinetics and penetration of continuous infusion topotecan in children with central nervous system tumors. Cancer Chemother Pharmacol, 1996. 37(3): p. 195-202.

166. Turner, P.K., et al., Development and validation of limited sampling models for topotecan lactone pharmacokinetic studies in children. Cancer Chemother Pharmacol, 2006. 57(4): p. 475-82.

167. Zamboni, W.C., et al., Phenytoin alters the disposition of topotecan and $N$-desmethyl topotecan in a patient with medulloblastoma. Clin Cancer Res, 1998. 4(3): p. 783-9.

168. Shen, J., et al., Compartment-specific roles of ATP-binding cassette transporters define differential topotecan distribution in brain parenchyma and cerebrospinal fluid. Cancer Res, 2009. 69(14): p. 5885-92.

169. Carcaboso, A.M., et al., Tyrosine kinase inhibitor gefitinib enhances topotecan penetration of gliomas. Cancer Res, 2010. 70(11): p. 4499-508. 
170. Leu, A.J., et al., Absence of functional lymphatics within a murine sarcoma: a molecular and functional evaluation. Cancer Res, 2000. 60(16): p. 4324-7.

171. Ferrari, A., et al., Soft tissue sarcoma across the age spectrum: a population-based study from the Surveillance Epidemiology and End Results database. Pediatr Blood Cancer, 2011. 57(6): p. 943-9.

172. Meza, J.L., et al., Analysis of prognostic factors in patients with nonmetastatic rhabdomyosarcoma treated on intergroup rhabdomyosarcoma studies III and IV: the Children's Oncology Group. J Clin Oncol, 2006. 24(24): p. 3844-51.

173. Hsu, J.Y. and H.A. Wakelee, Monoclonal antibodies targeting vascular endothelial growth factor: current status and future challenges in cancer therapy. BioDrugs, 2009. 23(5): p. 289-304.

174. Goulart, B. and S. Ramsey, A trial-based assessment of the cost-utility of bevacizumab and chemotherapy versus chemotherapy alone for advanced non-small cell lung cancer. Value Health, 2011. 14(6): p. 836-45.

175. McGonigle, K.F., et al., Combined weekly topotecan and biweekly bevacizumab in women with platinum-resistant ovarian, peritoneal, or fallopian tube cancer: results of a phase 2 study. Cancer, 2011. 117(16): p. 3731-40.

176. Neale, G., et al., Molecular characterization of the pediatric preclinical testing panel. Clin Cancer Res, 2008. 14(14): p. 4572-83.

177. Smith, M.A. and B.D. Anderson, A window on reality? J Clin Oncol, 2004. 22(8): p. 1360-2.

178. Zhuang, Y., et al., Topotecan central nervous system penetration is altered by a tyrosine kinase inhibitor. Cancer Res, 2006. 66(23): p. 11305-13.

179. Leggas, M., et al., Microbore HPLC method with online microdialysis for measurement of topotecan lactone and carboxylate in murine CSF. J Pharm Sci, 2004. 93(9): p. 2284-95.

180. Furman, W.L., et al., Escalating systemic exposure of continuous infusion topotecan in children with recurrent acute leukemia. J Clin Oncol, 1996. 14(5): p. 1504-11.

181. D'argenio DZ, S.A., ADAPT IV user's guide: pharmacokinetic/pharmacodynamic systems analysis software. Los Angeles: Biomedical Simulations Resource, 2009.

182. Ulahannan, S.V. and J.R. Brahmer, Antiangiogenic agents in combination with chemotherapy in patients with advanced non-small cell lung cancer. Cancer Invest, 2011. 29(4): p. 325-37.

183. Miller, V.A., et al., Pilot trial of the epidermal growth factor receptor tyrosine kinase inhibitor gefitinib plus carboplatin and paclitaxel in patients with stage IIIB or IV non-small-cell lung cancer. J Clin Oncol, 2003. 21(11): p. 2094-100.

184. Potmesil, M., Camptothecins: from bench research to hospital wards. Cancer Res, 1994. 54(6): p. 1431-9.

185. Pommier, Y., et al., Cellular determinants of sensitivity and resistance to DNA topoisomerase inhibitors. Cancer Invest, 1994. 12(5): p. 530-42.

186. Pommier, Y., DNA topoisomerase I and II in cancer chemotherapy: update and perspectives. Cancer Chemother Pharmacol, 1993. 32(2): p. 103-8.

187. Zamboni, W.C., et al., Pharmacokinetics (PK) of topotecan (TPT) in pediatric patients with normal and altered renal function. Proc Am Soc Clin Oncol, 1996. 15(Abstr 371): p. 180. 
188. Tian, Q., et al., Topotecan is a substrate for multidrug resistance associated protein 4. Curr Drug Metab, 2006. 7(1): p. 105-18.

189. Stopeck, A., et al., Results of a Phase I dose-escalating study of the antiangiogenic agent, SU5416, in patients with advanced malignancies. Clin Cancer Res, 2002. 8(9): p. 2798-805.

190. Kitamura, R., et al., Decrease in plasma concentrations of antiangiogenic agent TSU-68 ((Z)-5-[(1,2-dihydro-2-oxo-3H-indol-3-ylidene)methyl]-2,4-dimethyl-1H pyrr ole-3-propanoic acid) during oral administration twice a day to rats. Drug Metab Dispos, 2007. 35(9): p. 1611-6.

191. Kitamura, R., et al., Time-dependent induction of rat hepatic CYPIAl and CYP1A2 expression after single-dose administration of the anti-angiogenic agent TSU-68. Drug Metab Pharmacokinet, 2008. 23(6): p. 421-7.

192. Shukla, S., et al., Sunitinib (Sutent, SU11248), a small-molecule receptor tyrosine kinase inhibitor, blocks function of the ATP-binding cassette (ABC) transporters P-glycoprotein (ABCB1) and ABCG2. Drug Metab Dispos, 2009. 37(2): p. 359-65.

193. Reed, B.Y., et al., Angiogenic growth factors correlate with disease severity in young patients with autosomal dominant polycystic kidney disease. Kidney Int, 2011. 79(1): p. 128-34.

194. Willett, C.G., et al., Direct evidence that the VEGF-specific antibody bevacizumab has antivascular effects in human rectal cancer. Nat Med, 2004. 10(2): p. 145-7.

195. Jain, R.K., R.T. Tong, and L.L. Munn, Effect of vascular normalization by antiangiogenic therapy on interstitial hypertension, peritumor edema, and lymphatic metastasis: insights from a mathematical model. Cancer Res, 2007. 67(6): p. 2729-35.

196. Hu-Lowe, D.D., et al., Targeting activin receptor-like kinase 1 inhibits angiogenesis and tumorigenesis through a mechanism of action complementary to anti-VEGF therapies. Cancer Res, 2011. 71(4): p. 1362-73.

197. Finco, D., et al., Comparison of competitive ligand-binding assay and bioassay formats for the measurement of neutralizing antibodies to protein therapeutics. $\mathrm{J}$ Pharm Biomed Anal, 2011. 54(2): p. 351-8.

198. Zhong, Z.D., et al., Identification and inhibition of drug target interference in immunogenicity assays. J Immunol Methods, 2010. 355(1-2): p. 21-8.

199. Ma, J. and D.J. Waxman, Dominant effect of antiangiogenesis in combination therapy involving cyclophosphamide and axitinib. Clin Cancer Res, 2009. 15(2): p. 578-88.

200. Troiani, T., et al., Investigation of two dosing schedules of vandetanib (ZD6474), an inhibitor of vascular endothelial growth factor receptor and epidermal growth factor receptor signaling, in combination with irinotecan in a human colon cancer xenograft model. Clin Cancer Res, 2007. 13(21): p. 6450-8.

201. Kasman I, et al., Mechanistic evaluation of the combination effect of anti-VEGF and chemotherapy. AACR 99th annual meeting, 2008: p. A2494. 


\section{VITA}

Zaifang Huang was born in 1980 in P. R. China. She received her bachelor degree in Pharmacy and Master of Science in pharmaceutical science from Zhejiang University. She entered the Pharmaceutical Sciences Graduate Program with a major in pharmaceutics at the College of Pharmacy, University of Tennessee Health Science Center in August 2009. She was accepted into the lab of Dr. Clinton F. Stewart in Department of Pharmaceutical Science in St. Jude Children's Research Hospital. She expects to graduate in December 2011 with the degree of Master of Science in Pharmaceutical Sciences. 